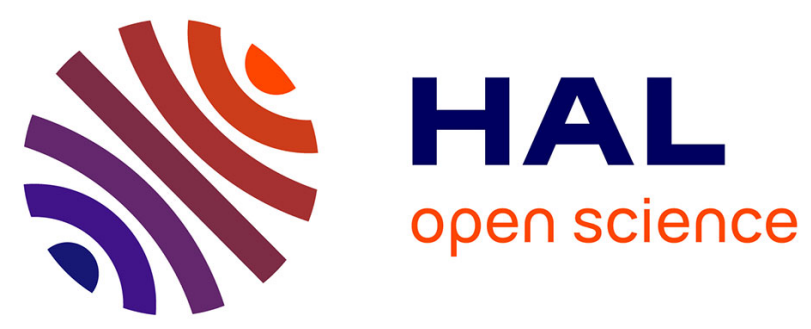

\title{
La création de la Mission archéologique française en Albanie (1922-1923), entre Armée d'Orient et modèles institutionnels
}

Annick Fenet

\section{- To cite this version:}

Annick Fenet. La création de la Mission archéologique française en Albanie (1922-1923), entre Armée d'Orient et modèles institutionnels . A. Fenet; N. Lubtchansky. Pour une histoire de l'archéologie XVIIIe siècle-1945. Hommage de ses collègues et amis à Ève Gran-Aymerich, 5, Ausonius, p. 419-446, 2015, Scripta Receptoria. halshs-01566865

\section{HAL Id: halshs-01566865 \\ https://shs.hal.science/halshs-01566865}

Submitted on 21 Jul 2017

HAL is a multi-disciplinary open access archive for the deposit and dissemination of scientific research documents, whether they are published or not. The documents may come from teaching and research institutions in France or abroad, or from public or private research centers.
L'archive ouverte pluridisciplinaire HAL, est destinée au dépôt et à la diffusion de documents scientifiques de niveau recherche, publiés ou non, émanant des établissements d'enseignement et de recherche français ou étrangers, des laboratoires publics ou privés. 


\title{
Pour une histoire de l'archéologie XVIII ${ }^{\mathrm{e}}$ siècle - 1945
}

Hommage de ses collègues et amis à Ève Gran-Aymerich

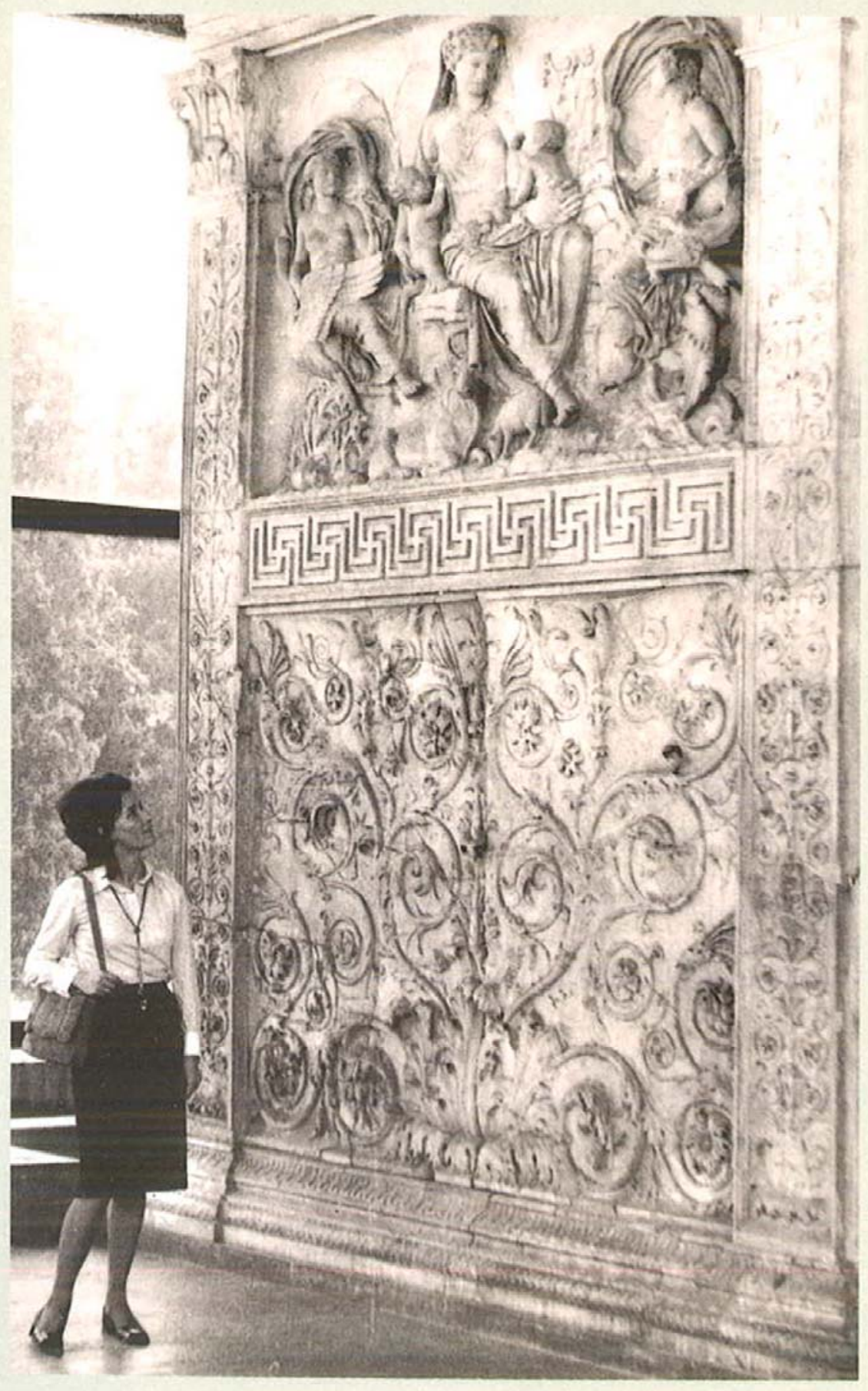

\author{
textes réunis par \\ Annick FENET \\ et Natacha LUBTCHANSKY
}

ScriptaReceptoria ${ }^{5}$ 


\section{Pour une histoire de l'archéologie XVIII ${ }^{\mathrm{e}}$ siècle - 1945}




\section{Annick Fenet}

est historienne et archéologue, membre d'AOROC (UMR 8546, ENS-

CNRS)

\section{Natacha Lubtchansky}

est Professeur d'archéologie et d'histoire de l'art classique à l'Université François-Rabelais de Tours ; E.A. 6298 - Centre Tourangeau d'Histoire et d'études des Sources (CeTHiS)

Illustration de couverture :

Visite de l'Ara Pacis (Rome, 1971).

(c) Cl. Jean Gran-Aymerich 
Ausonius Éditions

- Scripta Receptoria 5 -

\title{
Pour une histoire de l'archéologie XVIII ${ }^{\mathrm{e}}$ siècle - 1945
}

\author{
Hommage de ses collègues et amis \\ à Ève Gran-Aymerich
}

\author{
textes réunis par Annick Fenet \\ \& Natacha Lubtchansky
}

Cet ouvrage a été publié avec le soutien du laboratoire d'excellence TransferS (programme Investissements d'avenir ANR-10-IDEX-ooor-02 PSL* et ANR-10-LABX-oog9), du laboratoire AOROC (ENS-CNRS),

de l'Université François-Rabelais (Tours) et du CeTHiS (EA 6298) 


\section{Notice catalographique :}

Fenet, A. et N. Lubtchansky, éd. (2015) : Pour une histoire de l'archéologie XVIII siècle-1945, Hommages de ses collègues et amis à Ève Gran-Aymerich, Ausonius Scripta Receptoria 5, Bordeaux.

\section{Mots clés :}

Archéologie, historiographie, Ève Gran-Aymerich, sciences de l'Antiquité, préhistoire, transferts culturels, Europe, $\mathrm{XIX}^{\mathrm{e}}$ et $\mathrm{XX}^{\mathrm{e}}$ siècles, mouvement des Lumières, patrimoine, orientalisme, histoire de l'enseignement

\section{AUSONIUS}

Maison de l'Archéologie

F - 33607 Pessac cedex

http://ausoniuseditions.u-bordeaux-montaigne.fr

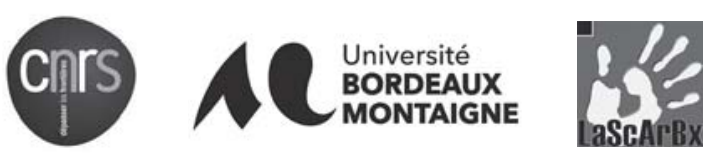

Directeur des Publications : Olivier Devillers

Secrétaire des Publications : Julie Charlet

Graphisme de Couverture : Julie Charlet

Tous droits réservés pour tous pays. La loi du 11 mars 1957 sur la propriété littéraire et intellectuelle interdit les copies ou reproductions destinées à une utilisation collective. Toute représentation ou reproduction intégrale ou partielle faite par quelque procédé que ce soit sans le consentement de l'éditeur ou de ses ayants droit, est illicite et constitue une contrefaçon sanctionnée par les articles 425 et suivants du Code pénal.
(c) AUSONIUS 2015
ISSN : $2427-4771$
ISBN : 978-2-35613-131-7
Achevé d'imprimer sur les presses
de l'imprimerie Bonnin
Z.I. de Canéjan
14-16, rue Pierre Paul de Riquet
F - 33610 Canéjan
$1^{\text {er }}$ septembre 2015 


\section{Sommaire}

Annick Fenet et Natacha Lubtchansky, Introduction

Jürgen von Ungern-Sternberg (trad. française de Pascale Rabault-Feuerhahn)

Avant-propos : Notizen zu einer deutsch-französischen Zusammenarbeit

1. Aux origines de l'archéologie

Alain Schnapp, L'oubli et la redécouverte d'Olympie des origines à l'expédition de Morée.

La philosophie antique des ruines

Maria Bonghi Jovino, Pompei. Una grande "avventura" culturale dal Settecento alla metà del XX secolo

Mario Torelli, Andrea Lombardi. Un intellettuale meridionale e il mito degli Italici nell'Italia del Risorgimentale

Pierre Pinon, La découverte de la céramique sigillée gallo-romaine : une approche "moderne" dès les XVIII et XIX $X^{e}$ siècles

Guillermo Pérez Sarrión et Almudena Domínguez Arranz, Archéologie et modernité en Espagne au XVIII siècle. Grèce et Rome à l'époque des Lumières

Jorge Maier Allende, Continuidad y ruptura de la arqueología española en el Siglo de las Luces

Caterina Maderna, Franz I. von Erbach zu Erbach.

Zur Erziehung und Bildung eines aufgeklärten Antikensammlers im Odenwald

Pierre-Sylvain Filliozat, 1817-1821 : Les Monuments de l'Hindoustan de Louis-Mathieu Langlès

François Djindjian, Jean Potocki (1761-1815) : voyages, expéditions et archéologie.

Des Histoires anciennes aux Chronologies et au Mémoire de l'expédition en Chine 
2. Archéologie et transferts culturels

Suzanne Marchand, The Dialectics of the Antiquities Rush

Dominique Briquel, Les Sarcophages des Époux du Museum of Fine Arts de Boston ou une victoire des États-Unis d'Amérique sur la France de Napoléon

Stefan Rebenich, "Ich komme schwerlich wieder".

Theodor Mommsen und das Deutsche Archäologische Institut

Fernando Wulff Alonso, Juzgando a los pueblos desde Grecia y Roma. De la India a Iberia, pasando por la Galia

Bénédicte Savoy, Nefertiti and co. à Berlin, 1913-1925

Antoine Hermary, Marseille phénicienne : un mythe du XIX siècle

Jean Hadas-Lebel, Jules Martha et la controverse sur l'origine de l'étrusque

Margarita Díaz-Andreu, Les théories voyageuses :

l'accueil britannique réservé aux connaissances sur le Paléolithique nées en France au cours de la première moitié $d u x x^{e}$ siècle

Francisco Gracia-Alonso, La diffusion de la recherche archéologique espagnole en France. Raymond Lantier et les cours à l'École du Louvre, 1939-1943

Noël Coye, Préhistoire européenne et africaine : l'analogie typologique après l'abandon de la théorie du synchronisme (1900-1950)

3. Archéologie et institutions

Sandrine Maufroy, Un pionnier de l'archéologie moderne aux Pays-Bas :

Caspar Reuvens (1793-1835)

Martín Almagro Gorbea, El Gabinete de Antigüedades

de la Real Academia de la Historia en el siglo XIX

Natacha Lubtchansky, L'œil du savant : le cours sur l'art étrusque de Désiré Raoul-Rochette à la Bibliothèque du Roi (1828) 
Mercedes Volait, Une entreprise autodidacte aux premiers temps de l'archéologie égyptienne: "L'Égypte monumentale" d'Émile Prisse d'Avennes (1807-1879)

Charles Guittard, Émile Masqueray et l'Algérie:

le regard d'un historien humaniste sur l'Algérie du XIX siècle

Hervé Duchêne, Salomon Reinach et George Balagny:

sur un épisode méconnu de l'histoire de la photographie française

Annick Fenet, La création de la Mission archéologique française en Albanie (1922-1923), entre Armée d'Orient et modèles institutionnels

Michel Dewachter, Historiographie ou archéologie de papier? Remarques à propos des archives de l'égyptologue Georges Legrain (1865-1917)

En guise de conclusion :

Houcine Jaïdi, L'archéologie tunisienne au lendemain de la Révolution du 14 janvier 2011. État des lieux, inquiétudes et horizons

Résumés

Index général 


\section{La création de la Mission archéologique française en Albanie (1922-1923), entre Armée d'Orient et modèles institutionnels}

Annick Fenet

Comme l'a montré Ėve Gran-Aymerich, le développement de l'archéologie française en Méditerranée n'a pas seulement suivi les exigences d'une science en évolution, mais aussi "le contexte politique et les intérêts de l'État à un moment précis de son histoire" ; il s'est par ailleurs opéré à l'aide de modèles institutionnels reproduits et adaptés à ces conditions'. L'ouvre réalisée en Albanie à partir de 1923, grâce à une convention signée entre les deux pays, en constitue un bon exemple. Si les travaux scientifiques accomplis dans ce cadre par Léon Rey (1887-1954) et poursuivis encore aujourd'hui par la Mission archéologique et épigraphique française en Albanie ${ }^{2}$ ont fait l'objet de nombreuses publications, nous nous attacherons ici à expliquer la genèse de l'accord diplomatique sous le deuxième gouvernement Poincaré (1922-1924), en reconstituant les différentes versions du texte, et à replacer dans leur contexte la nature des premiers travaux menés sur le terrain. Cette évocation de limbrication entre archéologie et politique dans l'entre-deux-guerres est rendue possible grâce aux Archives nationales en Île-de-France, à celles du ministère des Affaires étrangères (MAE $)^{3}$ et au journal de l'initiateur de la mission française dans la jeune Albanie indépendante, le député et futur ministre Justin Godart $(1871-1956)^{4}$, publié en langue albanaise et dont des extraits viennent d'être retraduits en français au sein d'une biographie de Léon Rey.

\section{LE PROJET DE GODART POUR L'ALBANIE}

Les liens de Godart avec l'Albanie remontent à la veille de la Grande Guerre, lorsqu'il fut membre de la Commission internationale d'enquête dans les Balkans (Serbie, Bulgarie, Grèce, Turquie, Albanie) organisée par la Dotation Carnegie pour la paix internationale en 1913 et conduite par le diplomate Paul d'Estournelles de Constant - qui avait lui-même déjà

EGA. 2 (1998) ; EGA. 65 (2000).

Relancée en 1992 par P. Cabanes et aux travaux de laquelle j'ai eu le plaisir de participer de 1994 à 2002 (exploration de la cité d'Apollonia, poursuivie aujourd'hui sous l'égide de J.-L. Lamboley, F. Quantin et S. Verger).

3 En revanche, parmi les archives Léon Rey léguées aux Archives nationales de Tirana, il ne semble s'y trouver aucun document relatif à la convention. Je remercie chaleureusement M. Jean Gabriel Rey, auteur de ce legs, d'avoir si obligeamment répondu à mes questions.

4 Ministre dans les gouvernements Herriot (du Travail, de l'Hygiène, de l'Assistance et de la Prévoyance sociale, du 14 juin 1924 au 17 avril 1925 ; de la Santé publique du 3 juin au 18 décembre 1932) et sénateur de 1926 à la Seconde Guerre mondiale (où il refuse les pleins pouvoirs à Pétain). 
participé à la mission de 1879 de la Commission de délimitation des frontières entre l'empire ottoman et les nouveaux territoires indépendants du Monténégro, de la Serbie et de la Bulgarie $^{5}$. Il s'agissait alors d'établir un rapport sur les violences commises contre les populations civiles lors des guerres balkaniques, entre Bulgares, Serbes et Grecs ${ }^{6}$. C'est dans ce contexte que l'Albanie proclama pour la première fois son indépendance, en novembre $1912^{7}$.

La Première Guerre mondiale et les années qui suivent voient le pays occupé par les Grecs, les Italiens, les Serbes, les Monténégrins et les Austro-hongrois ; les Français quant à eux occupent le bassin de Korça, établi en province autonome, et Shkodra. L'Albanie restant menacée de démantèlement, et ce malgré sa reconnaissance par la SDN le 17 décembre 1920, Estournelles de Constant propose à la Dotation Carnegie d'envoyer une nouvelle mission, conduite par son "ami" Justin Godart, dont il loue la droiture et la franchise ${ }^{8}$. Bien que profondément "philhellène" depuis le dernier quart du XIX ${ }^{\mathrm{e}}$ s. et partisan de Venizelos, ainsi qu'admirateur de certains politiciens serbes qu'il avait eu l'occasion de fréquenter, Estournelles de Constant condamne les guerres balkaniques et les malheurs subis par "la pauvre Albanie,

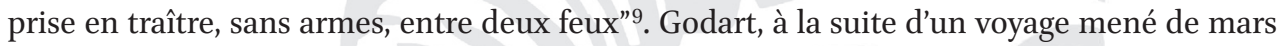
à mai 1921 (fig. 1), rédige un rapport qu'il publie en livre aux Presses universitaires de France, avec une préface de son ancien acolyte ${ }^{10}$, dans lequel il affirme, en s'appuyant notamment sur Gobineau et Victor Bérard, la réalité de la nation albanaise, existant depuis les temps antiques, et le bien-fondé de l'existence d'un État albanais. La question ethnique s'appuie sur l'identification d'une race illyrienne, orientale, reconnaissable d'ailleurs à des traits physiques bien spécifiques, et d'une "âme albanaise" qui a su résister aux Turcs" : "probablement la seule race entièrement autochtone subsistant dans les Balkans [qui,] dès le temps des Pélasges, puis des Illyriens, a su conserver dans ses montagnes la pureté de son sang, de ses mœurs et de sa langue"12. On notera à ce propos que de telles affirmations ont perduré tout au long du siècle et qu'elles ont constitué une antienne de la propagande nationale de l'Albanie d'Enver Hoxha et de Ramiz Alia (1944-1991). Dans le second semestre 1921, suite à la condamnation par la SDN des attaques serbes contre les populations albanaises, le nouvel État voit enfin les troupes étrangères quitter son territoire. Godart y retourne pour une nouvelle mission en mai 1922, toujours mandaté par la Dotation Carnegie. Désormais, il fait de l'Albanie son cheval de bataille, défendant le "petit" pays contre les grandes puissances, dans un esprit de politique internationale wilsonien et croyant en une paix mondiale obtenue par l'action de la SDN plutôt que par la diplomatie traditionnelle ${ }^{13}$. Y multipliant ses séjours, il tente de développer les collaborations entre la France et l'Albanie - la première a

Estournelles de Constant 1922, 1-16.

Franck 2004, 207-209.

Cabanes 1994, 62-64.

Franck 2004, 209 sq. ; Estournelles de Constant 1922, 22.

Estournelles de Constant 1922, 19-20.

Godart 1922. Ce rapport tient compte des événements qui ont eu lieu dans les mois qui ont suivi son retour en France.

Godart 1922, 33-46 ; Franck 2004, 210-211.

AMAE, Albanie 20 : rapport C202 de la Commission d'enquête de la SDN daté 19 avril 1922.

13 Franck 2004, 211-216. Cette troisième mission a donné lieu à un nouveau rapport de Godart, intitulé L'Albanie en 1922. 
en effet reconnu la seconde le 9 novembre 1921, en même temps que l'Angleterre, l'Italie et le Japon ${ }^{14}-$, sur tous les plans : politique, économique et culturel $^{15}$. Parmi les atouts que le jeune État peut valoriser figurent ses beautés naturelles et son originalité favorables à "un pays de tourisme fort attrayant" ; sans oublier ses richesses patrimoniales, auxquelles Godart consacre plus d'une page et sur lesquelles il achève son rapport de $1921^{16}$ :

"Sans tarder, l'Albanie doit inventorier et classer son patrimoine historique et archéologique. Des fouilles méthodiques ramèneront au jour des cités enfouies. Romains, Vénitiens et Turcs ont laissé dans le sol et sur le sol d'importants débris de leurs établissements. Et il est probable que la préhistoire trouverait, dans ce si vieux pays, des documents intéressants sur les origines de l'humanité. Pierres sculptées, inscriptions, monnaies, armes constitueraient une belle récolte d'antiques. (...) Il y a encore, debout, des monuments à conserver. Il en est qu'on devrait relever ou restaurer. Tout cela doit être entrepris au plus tôt. Un pays au riche passé comme l'Albanie se doit de recueillir et de mettre en ordre tout ce qui subsiste où un peu de son histoire est écrit, où un peu de l'âme des anciens s'est incorporé".

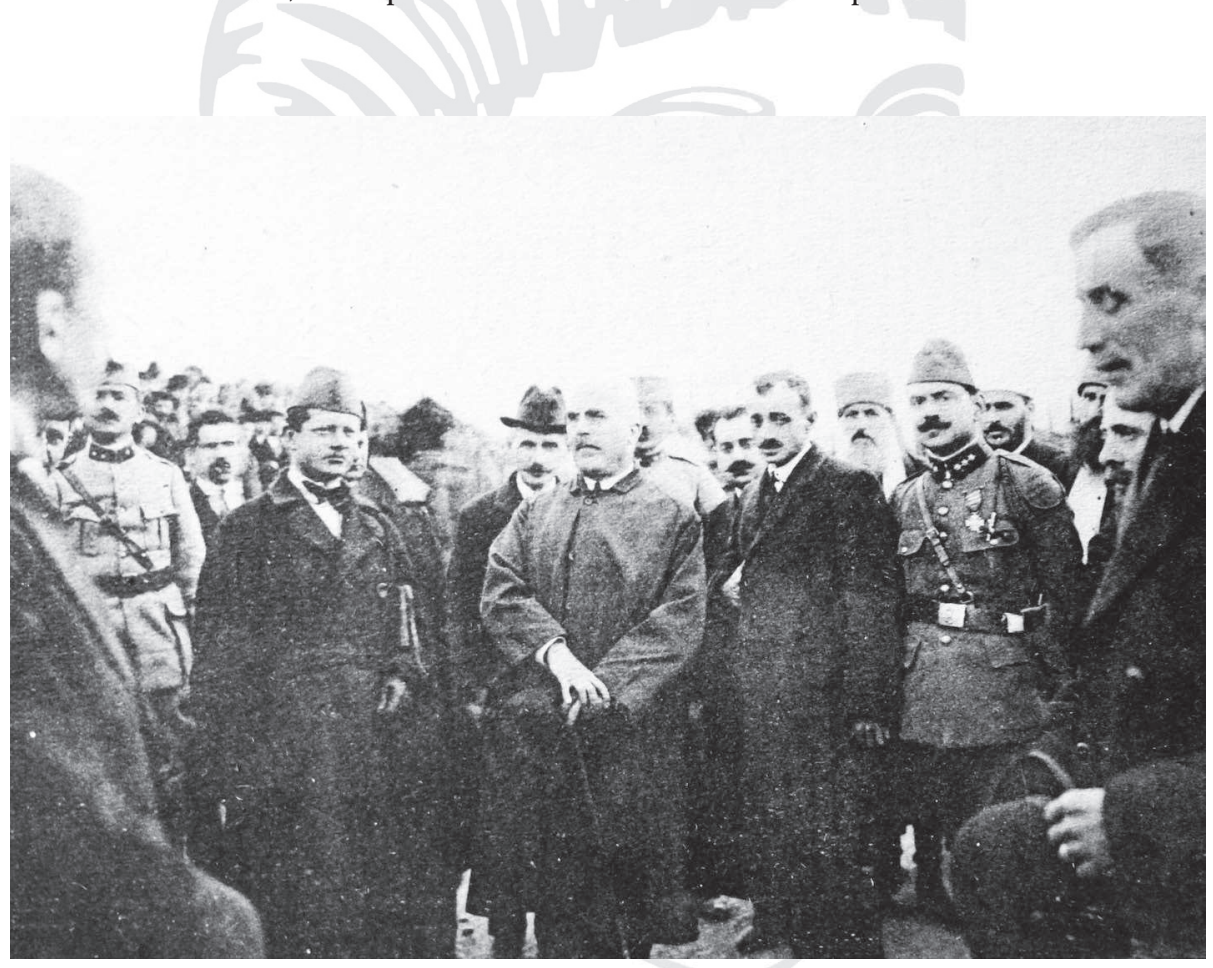

Fig. 1. Justin Godart lors de sa mission en Albanie de 1921 ; d'après Godart 1922, [pl. 17]. 
Amateur d'histoire régionale et sociale du Lyonnais pour la période révolutionnaire jusqu'au début $\mathrm{du} \mathrm{Xx}^{\mathrm{e}}$ s., collectionneur de vieux papiers, de médailles et d'objets folkloriques sur la capitale des Gaules, mécène du Musée historique de la ville et de musées de médecine ${ }^{17}$, Godart ne semble pas particulièrement intéressé par l'Antiquité ou le Moyen Âge. Pourtant, au cours de ses voyages, il a admiré la richesse archéologique du pays et décrit certaines villes et monuments : Durazzo (Durrës), des vestiges de la voie Egnazia, Bérat, Apollonia (Fieri et Poyani), Elbasan, sites dont il note les nombreux remplois de fragments antiques et les objets qui lui sont apportés par les habitants. Il déplore par ailleurs le "vandalisme" présent ou passé - notamment celui réalisé pendant la guerre par les Autrichiens - et appelle à une législation de protection du patrimoine national ${ }^{18}$.

Ce projet de valorisation patrimoniale de l'Albanie est proposé par le député à la fin de $1922^{19}$. Comme pour toute action française dans le domaine archéologique à l'étranger, l'idée doit être soumise aux départements compétents : au sein du ministère de l'Instruction publique (MIP), à la Direction de l'Enseignement supérieur qui gère la Commission des fouilles et à la tête de laquelle se trouve, de 1917 à 1926, Alfred Coville (1860-1942); ainsi qu'à la Sous-Direction de l'Europe aux Affaires politiques et commerciales du ministère des Affaires étrangères (MAE). Tout naturellement Léon Rey se trouve associé au projet. En effet, Godart et lui se sont connus au plus tôt pendant la guerre : une photo les montre dans la région de Salonique avec le général Sarrail (fig. 2). Le premier, alors sous-secrétaire d'État des services de santé militaire ( $1^{\mathrm{er}}$ juillet $1915-5$ février 1918 ), est venu y visiter les formations sanitaires de l'Armée d'Orient en août 1917 et à cette occasion s'est rendu sur les tumulus de Zeitenlik, qui ont été fouillés par Rey dans le cadre du Service archéologique de l'Armée d'Orient $(\mathrm{SAAO})^{20}$. Chartiste de formation - de même que Coville -, Rey a rejoint l'Armée d'Orient à l'automne 1915 avec le grade de maréchal des logis et a intégré son service archéologique. Officialisé en mai 1916, ce département militaire a été dissous en février 1919, mais son œuvre s'est poursuivie par le biais des troupes françaises restées dans les Balkans ${ }^{21}$.

Du côté albanais, le Gouvernement se trouve alors à Tirana, devenue capitale du pays, et se compose d'un Conseil de Régence créé en mars 1920 par l'assemblée de Lushnja et un Parlement élu depuis avril 1921. Pour les négociations, les Français auront affaire principalement à Ahmet Zogu, ministre de l'Intérieur - futur Président de la République puis roi Zog $\left(1925^{-1939}\right)^{22}$ - et au ministre de l'Éducation (ou Instruction publique) Rexhep Mitrovica. Le contexte politique est loin d'être serein : les années 1921-1924 sont marquées par une instabilité gouvernementale et des luttes entre les deux partis qui se partagent les sièges à l'Assemblée ; ainsi, les zoguistes sont mis en minorité lors des élections de l'automne 1923. Dans ces mêmes années, se pose pour la France le problème de défendre ses intérêts en Albanie où

Blazy 2004.

Godart 1922, 36-39 (Dürres), 66 (Egnazia), 71-74 (Bérat), 79-80 (Apollonia).

AN, F17-17225 : séance du 8 mars 1923.

20 Rama 2010, 26 et pl. 8 ; Rama 2012, 67. Voir les photos de la Médiathèque de l'architecture et du patrimoine : notamment la photo du 26 août 1917 (opérateur T. Dubray).

21 Homolle 1919 ; en dernier lieu (avec bibliographies) Adam-Veleni \& Koukouvou 2002, Farnoux 2012 et Fenet 2014.

22 Cabanes 1994, 64-67. 


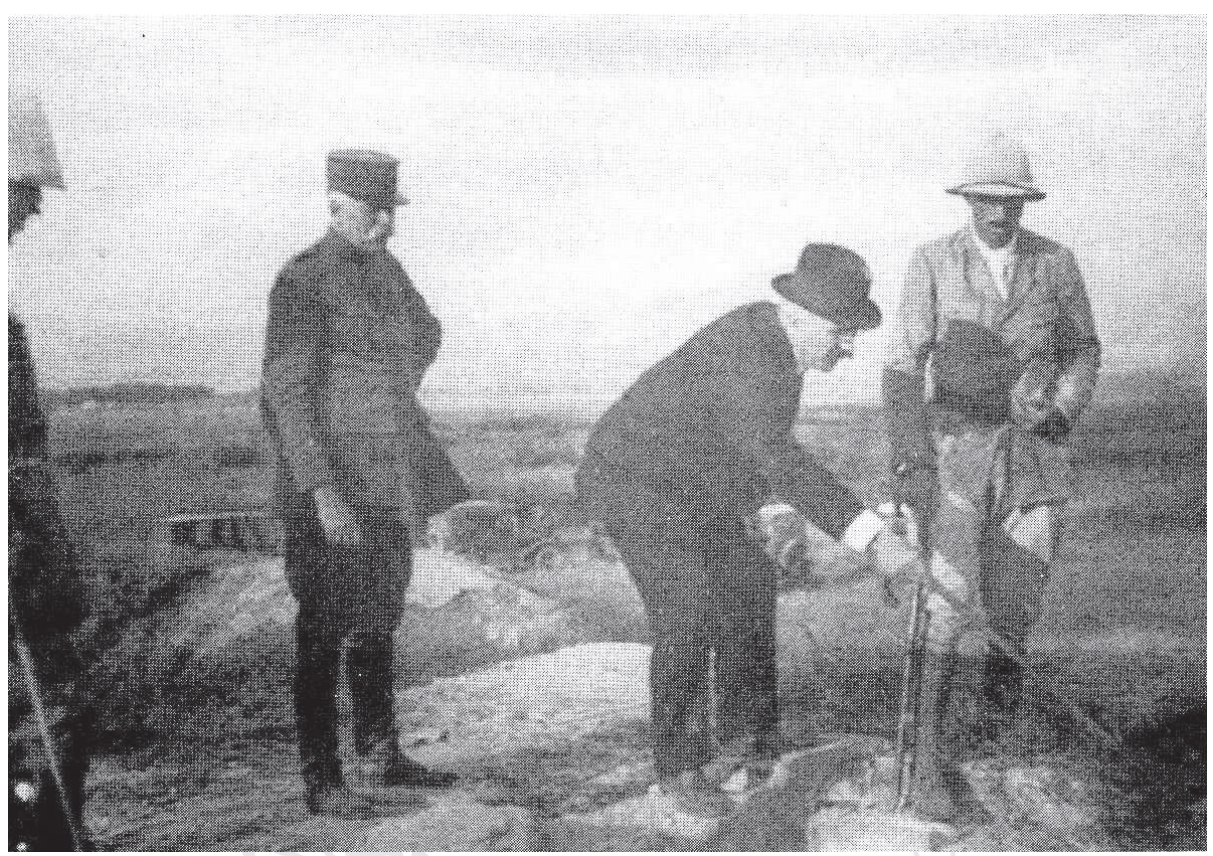

Fig. 2. Sarrail, Godart et Reyà Zeitenlik en 1917 ; d'après Rama 2010, 26 et pl. 8.

elle ne dispose que d'un chargé d'affaires, Jean Béguin-Billecocq (1875-1936), dont la résidence se trouve de surcroît à Scutari (Shkodra), et non pas à Tirana ou Durazzo où se règlent désormais les affaires ${ }^{23}$. Le diplomate connaît cependant bien le pays, où il œuvre depuis 1914, après avoir été drogman à Constantinople ; proche de Zogu, il obtiendra d'ailleurs de lui le titre de duc de Durazzo.

\section{SEPT MOIS DE NÉGOCIATIONS FRANCO-ALBANAISES}

Même si le MAE considérait que "la France [avait] développé avec ce pays de bonnes relations traditionnelles pendant la guerre", avec par exemple des cours de français ouverts à Korça par l'armée d'Orient ${ }^{24}$, et malgré les contacts privilégiés de Godart avec la jeune nation, les négociations ne se sont pas déroulées sans heurts. À la fin février ou dans les tout premiers jours de mars 1923, une réunion s'était tenue au MIP, au terme de laquelle il avait été décidé que Rey accompagnerait le député à Tirana pour réaliser la convention. Quelques jours plus tard, avant même qu'ils n'arrivent en Albanie, le compte-rendu qu'en donne Coville indique que des échanges avaient déjà eu lieu à ce sujet, vraisemblablement lors du précédent voyage du député :

“M. Godart a trouvé le Gouvernement albanais fort bien disposé à cet égard et la convention a de grandes chances d'aboutir, avec, toutefois, la réserve vraisemblable de voir attribuer [à 
d'au]tres que nous des sites, des champs de recherche, sur les points où durant cinq années consécutives la France n’aurait rien fait. (...) On ne sait pas encore avec certitude si le privilège qui nous est, en principe, réservé sera absolu ou non. (...)"25.

Les pourparlers commencent dès le lendemain de leur arrivée à Durrazzo, où les voyageurs observent les dernières trouvailles d'antiques ${ }^{26}$. À Tirana, Godart raconte :

“16 mars (...). L'après-midi j'ai avec le ministre [albanais] de l'Instruction publique Rexhep Mitrovica et Léon Rey un long entretien au sujet de la convention archéologique. J'abandonne le monopole, ce qui semble soulager de suite le ministre et vais, avec Rey, étudier les territoires sur lesquels portera la convention (...)".

Les deux Français travaillent les deux semaines suivantes à modifier le texte, en même temps que Godart multiplie les rendez-vous avec Ahmet Zogu et d'autres ministres albanais, cherchant d'autres coopérations dans tous les domaines possibles (pétrole, construction, éducation, santé, armée, etc.). Le 29 mars,

"Chez le ministre [des Affaires étrangères] Pandeli Evangjeli nous arrivons à la fin d'une scène violente entre Jella et Mitrovica au sujet de la convention archéologique. Jella reproche à ce dernier les tripotages de son frère. Pandeli en est suffoqué au point d'en avoir la parole coupée. À peine la retrouve-t-il qu'à mon tour je me plains violemment du retard apporté au vote de la convention (...). Rexhep Mitrovica vient à 10 havec la Convention archéologique. Il a besoin d'explications sur l'article 7 qui est cependant clair comme le jour. Il voudrait ajouter un article prévoyant un règlement à rédiger. Je refuse disant que la rédaction durerait des années. Il y aura un nouveau Conseil des ministres demain. J'ai manifesté ma colère de l'acte inamical pour la France qui consistait à retarder le vote de la convention. Il y a toujours grande effervescence au Parlement au sujet du retard apporté à nos affaires (...)"27.

La situation semble s'arranger puisqu'à leur retour, en avril 1923, un premier texte remanié est établi et transmis par Godart et Rey au MIP et au MAE (voir infra et annexe 1$)^{28}$.

Les deux hommes retournent en Albanie à la fin juillet. À la date du 2 août, Mitrovica leur demande encore des modifications qu'ils jugent confuses, tandis que Zogu sollicite la nomination d'un ministre de la République française à Tirana ${ }^{29}$. Vers le 14 août, à Paris, Godart se rend au MIP en compagnie de Rey pour accélérer la procédure, le Parlement albanais devant examiner le texte durant la session qui s'ouvre le 20 septembre suivant. Le texte, déposé dans une nouvelle version par l'archéologue, a donc fait encore l'objet de modifications la semaine précédente et l'on attend les observations du ministre de l'Instruction publique pour le transmettre d'urgence au MAE dans son dernier état. À la suite de cette visite, une nouvelle version du texte est transmise par Coville au Quai d'Orsay ${ }^{30}$; après quelques petites retouches, Béguin-Billecocq la signe le 28 août 1923 (voir infra et annexe 2$)^{31}$.

AN, F17-17225 : séance du 8 mars 1923.

AN, F17-17262, rapport de Rey du $1^{\text {er }}$ mai 1923 : départ de Paris le 6 mars, arrivée à Tirana le 15 mars ; Godart 2009, cité par Rama 2012, 29-30.

Godart 2009, cité par Rama 2012, 31-32.

AN, F17-17262 : rapports de Rey des $1^{\text {er }}$ mai et 22 sept. 1923.

Godart 2009, cité par Rama 2012, 36-39.

AN, F17-17262.

AMAE, Albanie 25 : télégramme du 28 août 1923 ; AN F17-17262 : lettre du MAE du 31 août 1923; AN, F17-17262 : rapport de Rey du 22 sept. 1923. 
Si les Français ont finalisé le texte, les négociations ne sont pas pour autant terminées. Le 8 septembre, il est approuvé en première lecture par le Parlement albanais, qui le renvoie pour examen à la Commission du MIP albanais ${ }^{3^{2}}$. Dans son journal, Godart, qui est revenu avec Rey à Tirana, raconte :

"Mercredi 12 sept. Nous achevons dans la matinée de rédiger contradictoirement au ministère la convention qui sera signée cet après-midi à 3 h. La carte est dressée et tapée à la machine. Rey arrive tout bouleversé d'un entretien avec le ministre [albanais] de l'Instruction publique qui, poussé par Patsch, l'organisateur autrichien, veut modifier la convention archéologique, restreindre nos terrains et déclare que telle quelle elle ne sera pas votée".

Son compatriote explique :

"Le 12 septembre je fus appelé par Redjeb Bey Mitrovitsa qui, cédant aux menées de M. Carl Patsch, 'organisateur' autrichien accrédité auprès du MIP [albanais], n'avait pas su défendre le texte qu'il avait signé. Il me fit part de nombreuses objections soulevées, disait-il, par les membres de la commission. La principale portait sur l'article 1 . Au lieu du privilège exclusif tel qu'il nous avait été accordé, on nous accordait un simple droit de priorité sur l'ensemble des terrains déjà choisis. Une modification aussi importante était inacceptable".

Godart poursuit :

"Rey doit le revoir cet après-midi pour lui dire notre avis. Je charge Rey de dire au Ministre que je ne changerai pas un mot de la convention sur laquelle avec lui, nous étions tombés d'accord et que j'ai fait signer par Poincaré. C'est maintenant une question de confiance entre France et Albanie et je n'admets pas que le gouvernement se dérobe. Rey porte cet ultimatum au ministre qui en est bouleversé. Il est le jouet de quelques intrigants et c'est un être sans caractère. J'ai à $7 \mathrm{~h}$ un entretien avec Ahmed [Zogu] sur ce point. Il semble un peu préoccupé, disant que sa majorité, par suite d'absences, n’est pas au complet. Il réunira demain un Conseil des ministres spécial. Pour le terrain nécessaire à la construction de la légation de France à Tirana il donnera toutes les facilités possibles".

Trois jours plus tard, la situation évolue favorablement, d'après Godart, en même temps que le règlement des tensions internes :

"15 sept., samedi. Eshref Frashëri est réélu président [du Parlement albanais]. Il est venu avant la séance dans ma loge pour me dire que la convention était acceptée par la commission. Que celle-ci a été unanime et que ce sera voté lundi par le Parlement. Sur les difficultés soulevées il passe vite, elles sont venues du ministre inspiré par Patch son conseiller autrichien (...)".

Pour Rey, ce succès est dû à "l'attitude énergique" de Godart et Béguin-Billecocq ${ }^{33}$.

Le député rentre en France, en ayant laissé l'affaire entre les mains de l'archéologue et du chargé d'affaires, non sans leur avoir recommandé de lâcher un peu de lest si nécessaire, mais de ne pas céder sur les points essentiels. Le 17 septembre, le texte est donc resoumis au Parlement, qui "accepte en principe certaines modifications proposées par les membres de l'opposition et auxquelles le gouvernement conservateur ne semblait pas hostile" et renvoie le vote au surlendemain. 
"Pour arriver à un accord, explique Rey, je proposai à Redjeb Bey Mitrovitsa la rédaction d'un paragraphe additionnel, dont M. le chargé d'affaires de France voulut bien approuver la teneur, et qui, susceptible de rallier la majorité de la Chambre, ne portait nullement atteinte aux principes essentiels de la convention. L'annexion de ce paragraphe m'a paru d'autant plus intéressante qu'il nous a permis de modifier l'article 4 en précisant le rôle des délégués du Gouvernement albanais" 34

Les modifications de dernière minute portent sur 6 articles $(3,4,6,7,14,15$ : voir infra Annexe 2). Après accord du ministre albanais et du chargé d'affaires, ce texte remanié est proposé au Parlement.

"Malgré l'opposition de certains députés, il allait être ratifié par la majorité des parlementaires, quand se produisit l'incident suivant. Le député de Scutari Louigi Gouracoutchi [sic] prit la parole et dit que le Gouvernement ne pouvait pas s'engager à envoyer auprès de la mission française des délégués albanais (art. 4) parce qu'il n'existait pas à l'heure actuelle en Albanie de personne capable de remplir ce poste. Le Ministre ne répondit point et devant le silence des partisans du Gouvernement, le Président de la Chambre Echref Fracheri suspendit la séance. Je me rendis immédiatement auprès de Redjeb Bey Mitrovitsa qui n'hésita point à partager l'avis du député de Scutari. Le chargé d'affaires de France étant survenu sur ces entrefaites, nous nous réunîmes dans le cabinet du Président. À cette réunion prirent part Mitrovitsa, Fracheri, et Ali Klissoura député et rapporteur de la convention. M. le chargé d'affaires exposa à ces messieurs tous les inconvénients qui résulteraient de la présence d'un savant étranger et peut-être allemand auprès du Directeur de la mission française. Le Président de la Chambre et le Ministre nous affirmèrent que dans le cas où nous maintiendrions notre point de vue la convention serait rejetée. J'eus alors l'impression d'une manœuvre de la dernière heure tentée par les éléments germanophiles qui, d'une manière plus ou moins occulte, font ici campagne contre toutes les concessions françaises. Le rejet pur et simple de la convention eût risqué de compromettre le résultat de tous nos efforts. Devant cette situation et la promesse formelle du gouvernement et d'Ali Klissoura un des leaders les plus en vue de l'opposition ${ }^{35}$, de ne pas désigner de délégués appartenant à une puissance ennemie de la France, M. Béguin-Billecocq a consenti à supprimer de le mot 'albanais', en faisant toute réserve, dans le cas où la promesse que le gouvernement venait de nous faire ne serait pas loyalement maintenue. La séance fut reprise et la convention votée à mains levées par la majorité des parlementaires" ${ }^{36}$.

L'interprétation du chargé d'affaires devant ce dernier rebondissement va dans le même sens :

"La ratification de notre convention a donné lieu à une âpre discussion à la Chambre albanaise et a même mis, un moment, le cabinet en échec. L'opposition et les éléments austroallemands, qui gravitent autour du Gouvernement et des Députés, ont tout tenté pour faire échouer cet accord en l'utilisant comme arme à la fois contre le Ministère Ahmed Zogu et contre l'influence française. Il s'est produit en pleine séance un sérieux incident (...) [à propos de l'article 4]. N'ayant pas le temps de demander des instructions (...) car toute remise aurait mis en danger la réussite de cette affaire, j'ai dû prendre sur le champ une décision

AN, F17-17262 : lettre au MIP du 22 sept. 1923. Eshref Frasheri (?-1938) et Ali Këlcyra (1891-1863) sont tous deux membres du parti de Fan Noli, adversaire de Zogu.

6 AN, F17-17262 : lettre de Rey au MIP du 22 sept. 1923. 
qui a assuré le vote de la convention. Depuis la ratification, (...) de nombreuses difficultés, vraisemblablement suscitées par le Dr Patsch, l'organisateur autrichien des Musées albanais, ont surgi pour la publication du décret (...) nécessaire pour permettre à M. Rey de commencer ses travaux. Je suis intervenu auprès du Président du Conseil et du Ministre des Affaires étrangères [albanais] (...)"37.

Coville, qui résume la modification lors de la séance de la Commission des missions du 23 octobre 1923, estime cependant qu'elle "peut avoir de lourdes conséquences" : "on peut avoir quelques inquiétudes, d'autant plus que le Gouvernement albanais a auprès de lui un conseiller technique qui est autrichien" ${ }^{3}$.

Après validation par le Parlement le 20 septembre et par le Haut Conseil d'État albanais, le journal officiel albanais publie le 11 octobre 1923 le décret du Conseil de Régence relatif à la convention archéologique. L'exemplaire de la convention conservée dans les archives albanaises est signé par le Premier ministre albanais et ministre de l'Intérieur Ahmet Zogu et par le ministre de l'Éducation Rexhep Mitrovica, et du côté français par le chargé d'affaire ${ }^{39}$. Les archives françaises concernant l'Albanie que nous avons consultées aux Archives nationales et au MAE ne conservent pas copie de l'accord final, mais des textes intermédiaires. Le texte français signé présenté en Annexe 2 est reconstitué à partir de la dernière version validée par les ministères parisiens en août 1923, complétée des ajouts portés à Tirana par Léon Rey avec l'aval du chargé d'affaires les 18 et 19 septembre 1923, et en comparaison avec la version albanaise publiée dans le Fletorja Zyrtare du 11 octobre 1923.

Du MODÈLE AFGHAN À UNE CONVENTION FRANCO-ALBANAISE : L'INFLUENCE FRANÇAISE

Si l'on peut ainsi suivre l'évolution des négociations en 1923, quel est donc le texte qui en a servi de support? C'est Coville qui nous donne la réponse :

"Dans les derniers mois de 1922, nous avons été avisés que M. Justin Godart, député du Rhône, trait d'union entre la France et l'Albanie, projetait de faire dans ce pays quelque chose d'analogue à ce que nous venions d'obtenir en Afghanistan : un projet de convention a été établi, calqué pour ainsi dire sur celui qu'avait préparé M. Foucher" ${ }^{40}$.

Godart a donc été inspiré par une convention archéologique contemporaine : celle réalisée et signée par l'indianiste et spécialiste de l'art du Gandhâra Alfred Foucher (1865-1952) à Kaboul le 9 septembre 1922. Le Lyonnais n'a en rien participé à cette aventure, mais il en a été informé très vraisemblablement entre juillet et décembre 1922, lorsque le projet de loi en vue de la création d'une légation française dans la capitale afghane a été présenté et adopté à la Chambre des députés ; il a alors été amplement question de l'accord scientifique qui venait d'être conclu ${ }^{41}$. Celui-ci fut lui-même conçu sur le modèle de la convention franco- 
perse de 1895 revue en 1900, avec des variantes voulues par l'indianiste qui, avant son entrée en Afghanistan en mars 1921, avait passé neuf mois en Perse et avait eu l'occasion d'observer la situation archéologique du pays ${ }^{42}$. Le texte finalisé en 13 articles par Foucher, seul interlocuteur français sur place à Kaboul et sans contact direct avec Paris, ne satisfaisait pas son auteur qui avait dû céder devant l'émir et ses ministres. Il y déplorait notamment la clause d'exclusivité, qui accordait aux seuls Français de fouiller en Afghanistan, alors que l'archéologue souhaitait partager ses recherches sur le terrain avec son ami le célèbre explorateur Aurel Stein (1862-1943), Hongrois naturalisé Britannique.

Le texte de la convention afghane a donc inspiré Godart, qui a dû le reprendre intégralement pour l'Albanie ; ce texte, qui a ainsi servi de base aux discussions de mars 1923, a d'abord été modifié comme on l'a vu en avril 1923 (Annexe 1). Si l'on compare cette deuxième version de la convention albanaise avec la convention afghane, on constate qu'elle a dans les grandes lignes - y compris dans la terminologie - respecté son modèle, en changeant l'entité "Gouvernement afghan" (ou "Gouvernement de Sa Majesté" [l'Émir]) en “Gouvernement albanais". L'article 1 du modèle, qui soulignait "le développement particulier pris en France par la science", devient plus précisément dans le cas présent "la compétence particulière des représentants de l'archéologie en France". Les restrictions religieuses et la notion de "respect" de la culture locale de l'article 2 sont strictement identiques à celles des conventions perse et afghane, avec l'ajout de la mention des édifices chrétiens, le peuple albanais étant composé d'Orthodoxes, de Catholiques et de Musulmans (en partie Bektashis).

On notera cependant que les Français ont pris soin de supprimer l'article 11 de la convention afghane, qui stipulait que "Le Gouvernement de Sa Majesté se réserve le droit de concéder à des savants étrangers la permission de faire des fouilles sur les sites où la Délégation scientifique française ne travaille pas et n'a pas l'intention de travailler avant l'expiration d'un certain délai, lequel ne saurait excéder cinq ans. Le Gouvernement afghan se concertera à ce sujet avec le Gouvernement français". Cette clause, qui restreignait l'exclusivité accordée à la France, avait été rajoutée par les Afghans, au grand dam de Foucher qui n’a pas manqué de commenter ce paragraphe auprès de ses autorités de tutelle ${ }^{43}$. Elle revient cependant sous une autre forme dans le texte final albanais, avec les articles 12 et 15. Les Français y ont par ailleurs renforcé l'article 8 , de façon à leur garantir la protection de leur droit scientifique vis-à-vis de leurs découvertes.

Les différences par rapport au modèle afghan, dans la $2^{\mathrm{e}}$ version et plus encore dans l'accord signé, révèlent les concessions qu'ont dû faire Godart et Rey lors des pourparlers et les points sensibles de l'état d'esprit des Albanais. Avant tout, plusieurs des modifications et ajouts montrent l'attachement de ces derniers à la notion de territoires et de limites des terres - ce qui est tout à fait compréhensible pour ce jeune État qui avait dû se défendre pour une délimitation de ses frontières, qui d'ailleurs n'étaient pas encore entièrement fixées à cette époque $\mathrm{e}^{44}$-, ainsi qu'à la propriété foncière et immobilière (art. 3 et 10). Le principal

42 Olivier-Utard [1997] 2003, 35-39 et Chevalier 2002, 355-359, 512-513, 515-516, 556-557 (textes des conventions perse et afghane) ; Fenet 2010, passim et 583-600 ; Fenet 2011b.

43 Fenet 2010, 21, 232-233, 237, 240, 325, 569, 596-60o.

44 Le protocole final de délimitation de la frontière serbo-albanaise fut signé le 27 janvier 1925 et la fixation de toutes les frontières reconnue le 30 juillet 1926 (AMAE, Albanie 1 : rapport du $1^{\mathrm{er}}$ mars 1927). 
changement consiste dans le territoire concerné, qui ne correspond pas à l'intégralité du pays, mais à une partie strictement définie (art. 1). L'article 12 final, totalement inédit, restreint encore les "zones" à fouiller à l'intérieur de ces terres.

Dans la deuxième version, la clause du partage restait presque identique à celle établie pour la Perse et l'Afghanistan : les archéologues français pouvaient emporter la moitié du matériel archéologique, à l'exception des objets en métaux précieux ou des "objets uniques" en autres matériaux - concept qui posa pourtant des tracas lors de la répartition des trouvailles afghanes ${ }^{45}$. Elle disparait du texte de septembre 1923 (art. 6-7), qui autorise seulement l'octroi à la France de "la moitié des objets en double", ce qui limite fortement les occurrences. En cela, cette règle a sans doute suivi la législation en vigueur en Grèce, où la convention de 1891 négociée pour la fouille de Delphes rendait la nation hellénique propriétaire de tout le matériel issu des fouilles - y compris les "doubles" ${ }^{46}$ - et la loi du 27 juillet 1899 interdisait toute exportation d'œuvres anciennes, ainsi que le règlement ottoman sur les antiquités et fouilles de $1906^{47}$. Dans la pratique, Léon Rey a laissé à la fin de chaque mission un enregistrement dûment établi des trouvailles laissées aux autorités albanaises ${ }^{48}$. L'ajout de l'ivoire parmi les matériaux précieux peut s'expliquer par plusieurs découvertes récentes dans le monde grec : par exemple à Mycènes ${ }^{49}$.

L'article 14 stipule que "les fouilles seront exécutées d'après les règles scientifiques" : cette formulation fait suite à un refus ferme de Paris (conjointement MIP et MAE) sur celle voulue par les Albanais, selon laquelle les fouilles seraient exécutées "conformément à un règlement élaboré d'un commun accord entre la Mission française et le Gouvernement albanais”, et supprimée du texte à la fin août $1923^{50}$. Cette modification tardive explique sans doute cette clause curieuse et quelque peu en contradiction avec la "compétence" archéologique de l'article 1. Peut-être en réponse à ce véto, un autre article a suscité les tensions les plus vives dans les derniers temps de la négociation : l'article 4, sur la nationalité des "savants" adjoints aux Français. Le texte publié dans le Journal officiel albanais indique, de façon volontairement ambiguë, "aqë dijetar e delegaten të Shtetit Shqiptarë" (autant de savants délégués de l'État albanais) ; on ne parle pas d'étrangers, mais de personnalités, sans précision de pays d'origine - à la différence des ouvriers de l'article 9 -, désignées par le Gouvernement de Tirana.

La convention signée met finalement davantage de pression sur les Français qu'en Perse et Afghanistan : obligation de fouiller et de publier vite (art. 8 et 15), charges financières des

45 Fenet 2010, $\mathrm{n}^{\circ} 248, \mathrm{n}^{\circ} 310$ ("un objet entièrement nouveau et dont le pareil n'est connu par aucune publication ni exposé dans aucun Musée") et p. 489, 599-6oo.

46 Sur cette négociation qui dura 10 ans : Amandry 1992 ; Dassios, 1992 (avec texte de la convention en 8 articles à la p. 142). Dans la genèse de cet accord (élaboré sur le modèle de la convention accordée aux Allemands pour Olympie en 1874), la clause concernant les trouvailles en double a été éliminée au début de 1887 pour aboutir en 1891 à l'art. 3, selon lequel “(...) la Grèce restera propriétaire de toutes les œuvres d'art, antiquités et tous autres objets dont la découverte serait due aux fouilles (...)”.

47 Art. XVI ; voir texte : Chevalier 2002, 500-505.

48 Par exemple, les listes signées du préfet de Durazzo pour un sondage de 1923 ou de Milto Sotir Gurra à la date du 31 août 1924 pour Apollonia (AN, F17-17262 ; Rama 2012, 62).

49 Cf. $\mathrm{BCH} 1922,494$.

$50 \quad$ AMAE, Albanie 25 : télégramme du 28 août 1923; AN F17-17262 : lettre du MAE du 31 août 1923. 
opérations (art. 3, 5, 10). Les Albanais se garantissent la propriété de leur patrimoine tant scientifique qu'immobilier, mais aussi les archives des travaux réalisés (art. 4, 5 et 8).

Du côté français, les négociations sont menées par Justin Godart et Léon Rey, c'est-à-dire des hommes n'appartenant pas au Quai d'Orsay. La III ${ }^{\mathrm{e}}$ République fait confiance et accorde des responsabilités aux hommes de science et de terrain dans le domaine des affaires étrangères et culturelles. Le processus est alors identique à celui observé en Afghanistan en 1922, lorsque Foucher, seul représentant du Gouvernement français à Kaboul, finalise le traité. Dans ce contexte de naissance de nouvelles nations, archéologie et processus diplomatique sont étroitement liés. Il convient dans les deux cas, pour le Gouvernement français, d'installer une ambassade à Tirana, comme il l'avait fait pour Kaboul. Il existe pourtant une différence notable, celle du calendrier : ici la signature de la convention (1923) précède le projet de création d'une légation dans la capitale. Pour que celle-ci aboutisse, il faut qu'un projet soit présenté et discuté à la Chambre des députés, avant validation éventuelle par le Sénat. Autre similitude entre l'Albanie et l'Afghanistan, le projet diplomatique est soutenu par des hellénistes qui exercent des mandats politiques : c'est le même Gaston Deschamps (18611931), qui avait déjà présenté le projet de loi en faveur de la légation afghane qui soutient le dossier albanais en $1924^{51}$. Cet ancien membre de l'ÉFA (promotion 1885), professeur au Collège de France et député des Deux-Sèvres (1919-1924), auteur de travaux portant sur la Grèce et le Proche-Orient, n'évoque cependant pas cette fois l'aspect archéologique, contrairement aux arguments avancés pour l'Afghanistan, mais invoque l'intérêt d'“un acte hautement amical d'où résultera pour notre influence dans les nouveaux États de l'Orient délivré un profil intellectuel et moral dont il est aisé d'apprécier toute la valeur" ${ }^{2}$.

En 1924, le projet n'est pas voté, pour des raisons financières et en raison de l'incertitude qui règne encore sur l'emplacement définitif de la capitale albanaise (à Scutari ou Tirana), mais il aboutira en 1927, en partie grâce à l'obstination de Godart ${ }^{53}$.

Les négociations, cela a été bien noté à l'époque, ont souffert de la présence de Carl Patsch (1865-1945), invité par le Gouvernement albanais à organiser le Musée national ${ }^{54}$. Celle-ci était également perçue comme une menace par le géologue Jacques Bourcart, qui séjourne plusieurs mois en Albanie durant l'année 1923 après y avoir œuvré pendant la guerre ${ }^{55}$. Ce qui est en jeu, c'est encore une fois l'influence française et l'opposition à la science germanique :

"En dehors du bénéfice que la science française doit retirer de la mission d'Albanie, notre pays y gagnera une influence d'autant plus importante que bien avant la guerre les Autrichiens et les Allemands briguaient le même privilège. La plupart des Albanais appartenant à la classe

51 AMAE, Albanie 1 : Chambre des députés, séance du 11 avril 1924, Projet de loi 6966, Imprimé 7595, Rapport du député G. Deschamps.

52 Fenet 2011 (avec extraits du rapport afghan du 30 nov. 1922, Document parlementaire $\mathrm{n}^{\circ} 5171$ ). Sur Deschamps : Jolly 1960-1977, t. IV, 1404-1405; Jamot 1933.

53 Voir historique rapporté par AMAE, Albanie 1, rapport $1^{\text {er }}$ mars 1927. Le premier ministre résident fut Gaston Moisson, baron de Vaux.

54 Clayer 2012.

55 AMAE, Albanie 1: note de Pieyre du 9 nov. 1923. Sur les travaux de Bourcart en Albanie, voir Papa 2000 (qui ne mentionne pas ce voyage). Il est l'auteur de deux ouvrages sur ce pays : Bourcart 1921 et Bourcart 1922. 
instruite ont reçu de Vienne ou de Berlin leur formation intellectuelle ; le contact entre cet élément de la population et les membres de la Mission ne peut être que favorable à notre pénétration dans le pays" ${ }^{56}$.

Coville "recommande" ainsi particulièrement à la Commission des missions celles d'Afghanistan et d'Albanie pour leur "caractère tout spécial d'intérêt scientifique et patriotique" 57 .

Cette rivalité, exacerbée par le conflit mondial, n'est pas la seule. Lors de la signature de la convention franco-afghane, les Anglo-Saxons avaient très vivement réagi tant sur le plan diplomatique que dans la presse. Malgré les restrictions portées dans la convention albanaise par rapport à son modèle afghan, le gouvernement américain n'est pas en reste, émettant le 18 mars 1924 - soit 10 mois après un premier communiqué officiel en juin-juillet 1923 à propos de l'Afghanistan - une protestation du conseiller de l'ambassade des ÉtatsUnis, $\mathrm{M}^{\mathrm{r}}$ Whitehouse, contre le monopole français des fouilles en Afghanistan et en Albanie, appuyée par le Président de l'Archaeological Institute of America Ralph V. D. Magoffin (de 1921 à 1931), par l'Institute et la plupart des musées américains. La réponse du Quai d'Orsay datée du 27 mars 1924 reste en substance la même que celle donnée l'année précédente, arguant que la France ne s'opposera pas à des demandes de fouilles dans ces pays émanant de "citoyens américains qui présenteraient toutes garanties requises au point de vue scientifique" ${ }^{8}$.

En ce qui concerne les Italiens, les relations s'avèrent également délicates. Dans l'été 1922, la presse italienne réagit de façon très critique au livre de Godart et condamne "la propaganda francese contro l'Italia in Albania" 59 . Face aux problèmes suscités par Patsch, les Français ont cependant tenté un rapprochement. Billecocq explique ainsi qu'étant donné ses bons rapports personnels [de Rey] avec le monde scientifique italien, je l'ai engagé à pressentir le Ministre d'Italie au sujet de la désignation par le Gouvernement albanais d'un savant italien comme délégué au Ministère albanais de l'Instruction publique auprès de lui. Le Marquis de Durazzo a accueilli ces ouvertures avec empressement ${ }^{\prime 60}$.

Les Italiens ne veulent cependant pas abandonner la partie : Roberto Paribeni, Directeur du Musée national romain et des Missions scientifiques italiennes dans le Levant propose à son ministère des Affaires étrangères de créer une mission italienne afin de concurrencer la France ; pour cela l'État italien envoie tout d'abord un jeune préhistorien, Luigi Ugolini (1895-1936), fouiller des tombes illyriennes dans le Nord de l'Albanie et prospecter dans la région de Vlora ${ }^{61}$. À partir de 1925, ce dernier entreprend quelques fouilles dans le sud du pays à Foiniké puis, à partir de 1928, il peut diriger une ambitieuse mission archéologique italienne dotée d'un solide programme de recherches de haut niveau et de moyens financiers considérables $^{62}$. Une concession de 30 ans, demandée par les Italiens dès décembre 1925, a

56 AN, F17-17262: note s.d.

57 AN, F17-17225 : séance du 8 mars 1923.

58 Fenet 2010, 125-127, 610 et $\mathrm{n}^{\circ} 101$; AMAE, Afghanistan 31.

59 Article de la Gazzetta di Venezia, 9 juillet 1922. AMAE 20 : lettre de l'ambassadeur à Rome au MAE du 2 août 1922.

$60 \quad$ AN, F17-17262 : lettre au MAE du 12 oct. 1923

61 Gilkes 2003-2004, 53-54.

62 Nallbani 2003-2004, 43. 
en effet été obtenue pour les préfectures d'Elbasan, Dibra, Kossova, Valona, Argyrocastro, Korytza et Tirana ; identique à celle des Français, elle est ratifiée par le Parlement albanais deux ans plus tard. Le ministre de la République française à Tirana, qui note alors, dans la publication d'un ouvrage sur les antiquités albanaises "l'omission volontaire des fouilles" de Léon Rey, conclut avec dépit : “On eût attendu d'un professeur réputé tel que M. Ugolini plus de sérénité scientifique et de loyauté” ${ }^{63}$.

\section{UNE MISSION HYBRIDE, EN PARTIE HÉRITIÈRE DE L'ARMÉE D'ORIENT}

Pour les Français, leurs prérogatives archéologiques en Albanie sont tout à fait légitimes. Après les premiers témoignages sur la région rapportés par des antiquaires, des voyageurs ou diplomates de diverses nationalités jusqu'au début du $\mathrm{XIX}^{\mathrm{e}}$ siècle ${ }^{64}$, ce sont des archéologues français qui, quelques décennies plus tard, y mènent quelques explorations et en ramènent des antiquités à Paris. Cet intérêt se place sous l'égide des grandes institutions françaises de l'époque : l'Académie des inscriptions et belles-lettres (AIBL) qui propose un sujet de recherche portant sur l'Épire en 1857 , le Louvre et l'École française d'Athènes (ÉFA) fondée en 1846. C'est ainsi que les Athéniens Xavier Gaultier de Claubry en 1859 puis Victor Bérard en 1890 décrivent certaines régions ou sites de l'Albanie et que Léon Heuzey, mandaté par Napoléon III, effectue une mission en Macédoine qui l'emmène en 1861 jusqu'à Apollonia d'Illyrie $^{65}$. Cette sorte de primauté historique française est soulignée par Léon Rey dans le discours qu'il prononce à Durazzo le 15 octobre, en guise d'inauguration des premiers coups de pioche ${ }^{66}$ : s'il ne dit pas un mot de la convention, il développe l'histoire de l'antique Épidamne-Dyrrachium, de ses remparts et de leur découverte ; en nommant Heuzey et son instigateur $^{67}$, il rattache implicitement la nouvelle mission aux expéditions impériales ou royales des XVIII ${ }^{\mathrm{e}}$ - XIX ${ }^{\mathrm{e}}$ s. (Égypte, Morée et Liban).

$\mathrm{Au}$ tournant $\mathrm{du} \mathrm{Xx}^{\mathrm{e}}$ siècle et durant ses deux premières décennies, paraissent des publications en langue allemande ou italienne sur le patrimoine archéologique de l'Albanie, notamment à la suite des expéditions menées par des Autrichiens ${ }^{68}$ et des Italiens à la faveur de la Grande Guerre. L'ÉFA reste cependant présente car elle souhaite étendre son champ d'investigation : vers l'Orient (au Proche-Orient et même en Asie centrale) et dans les Balkans, comme le montrent les papiers de son directeur Gustave Fougères (1913-1919) ${ }^{69}$. Ainsi, dès l'annexion par la Grèce de nouveaux territoires au Nord, celui-ci lance des campagnes d"exploration méthodique" menée par les Athéniens. Une mission est alors confiée en Épire à André Boulanger et Robert Lejeune au printemps 1914, mais auxquels "il est formellement interdit de se risquer dans la zone de l'Épire septentrionale actuellement contes-

63 AN, F17-17262 : copie de la lettre de la légation de France à Tirana à Briand du 6 déc. 1927.

64 Par exemple pour Apollonia : depuis Cyriaque d'Ancône en 1436 jusqu'au Britannique Leake et au Français Pouqueville (Dimo et al. 2007).

65 Cabanes 1996, 397-400.

66 AN, F17-17262 : rapport de la campagne 1923.

67 AN, F17-17262 : discours de Rey du 15 oct. 1923.

68 Voir bibliographies Münsterberg, Patsch et Praschniker in Fenet et al. 2007, xxviii-xxix.

69 Fenet 2013 (Moyen-Orient) ; Fenet 2011a (Asie Centrale). Cf. AEFA, Fonds C. Picard 2, 3, dossier "L'École d'Athènes". 
tée entre la Grèce et l'Albanie ; ils éviteront scrupuleusement les districts d'Argyrokastro [auj. Gjirokastër] et de Korissa [auj. Korçë] et toutes localités où sévit un état de guerre insurrectionnelle créant des risques particuliers" ${ }^{\prime 7}$. On connaît ensuite le rôle pris par l'École et Fougères dans l'œuvre de l'Armée d'Orient. Ce dernier écrit en 1916 :

“Du jour où les armes françaises furent attirées vers l'Orient hellénique, aux Dardanelles, en Troade, en Macédoine, l'École d'Athènes se trouvait prédestinée à apporter à nos armes le concours de sa connaissance du pays, du milieu et de la langue. (...) L'occupation de Salonique exerça une attraction qui a fini par se généraliser. L'Armée d'Orient ayant besoin d'officiers interprètes, de guides, d'archéologues, fit appel aux compétences d'autant plus empressées à se mettre à sa disposition qu'elles trouvaient là l'occasion de mettre utilement au service du devoir militaire leurs goûts et aptitudes professionnels"71.

Le service archéologique de l'Armée d'Orient aurait ainsi exploré, outre la Macédoine, les zones occupées par les forces françaises, dont l'Albanie où "en novembre 1917, [elle] avait reconnu 70 sites préhistoriques" ${ }^{\prime 2}$.

C'est pourquoi Léon Rey peut souligner, en 1923, la continuité scientifique de sa mission à travers l'objectif d'une "abondante moisson de documents propres à jeter une nouvelle lumière sur l'histoire des colonies grecques et romaines ainsi que sur les civilisations de l'antique Illyrie", en même temps que le voisinage et la parenté avec de telles institutions ("le peu de distances qui sépare les futurs chantiers albanais de nos écoles de Rome et d'Athènes est un avantage pratique qui s'ajoute encore à tout l'intérêt scientifique de la concession"73). La convention albanaise arrive également au moment où l'archéologie française en Thrace orientale, active depuis 1920 dans la continuité du SAAO, s'interrompt avec la signature du Traité de Lausanne qui détachait la Thrace orientale de la Grèce ${ }^{74}$. La mission en Albanie reste cependant indépendante de ces Écoles, l'École Française de Rome préférant privilégier dans les années 1920, "les champs de fouilles qui [lui] sont ouverts en Algérie" plutôt que les rivages balkaniques ${ }^{75}$. Les liens entre la mission albanaise et l'ÉFA s'établissent de façon indirecte : Léon Rey entretient d'excellents rapports avec le nouveau directeur de l'École, Charles Picard (1919-1925), son collègue de l'Armée d'Orient qui en 1922 a sollicité pour lui un prix de l'Académie en raison de ses travaux sur la Macédoine publiés dans le $B C H$ et à qui en retour le chartiste ne cache rien en début 1923 des projets albanais ${ }^{76}$. Par ailleurs, les Athéniens appartenant à la Commission du MIP (Théophile Homolle, Bernard Haussoullier, Edmond Pottier $)^{77}$ et à l'AIBL favorisent la mission de Léon Rey par l'attribution de financements. La Commission des fouilles archéologiques d'Albanie, dont l'existence est attestée avant la

CRAI 1915, 55. AEFA, fonds C. Picard 2,5 : fo 99, instructions du 16 avril 1914. AN, F17-13598 : rapport de Fougères daté 10 oct. 1916. AEFA, fonds C. Picard 32,8: $\mathrm{f}^{\circ}$ "Tépés et tumuli", s.d. AN, F17-17262 : note datée 19 août 1923. Son espoir de découverte d'une civilisation préhellénique en Albanie fut cependant déçu (Rama 2012, 58).

Marc 1997, 492-496. AN, F17-17262 : dossier "Correspondance 1924-1937". EGA. 65 (2000).

AEFA, Fonds C. Picard 3, 4 : lettre de Picard à Pottier du 20 juin 1922. Cabanes 1996, 400 : deux lettres de Rey à Picard, en janv. et avril 1923 - dont nous n'avons pas encore à ce jour trouvé trace dans les archives de l'ÉFA.

77 Sur la composition de cette Commission en 1923, voir Fenet 2010, 54, 617-618. 
signature de la convention, comporte très vraisemblablement également d'autres anciens membres de l'ÉFA, au premier chef Fougères ${ }^{78}$. Les deux hommes se connaissent depuis 1916 et ont travaillé ensemble notamment au musée de Salonique en 1917 ; le premier, qui souhaitait même voir le second régler l'organisation du SAAO, l'appelle "mon cher Maître" et continue après guerre à lui transmettre des documents scientifiques sur la Macédoine ${ }^{79}$.

Les moyens mis à disposition pour l'archéologie albanaise relèvent des mêmes combinaisons et systèmes que ceux avérés pour la mission afghane - en attendant un budget annuel spécifique et régulier qui viendra quelques années plus tard, à linstar de la Délégation archéologique française en Afghanistan (DAFA) ou de la Délégation archéologique française en Perse (DAFP). Lors de la séance du 8 mars 1923, la Commission des missions lui octroie une première subvention tout à fait respectable de 20000 francs (sur un total de crédits octroyés de 99 ooo $\mathrm{F}$ dont 25000 pour l'Afghanistan), pour une "mission qui ne saurait d'abord être que de prospection, le pays albanais pour la question qui nous occupe étant un pays absolument neuf ou presque, n'ayant été visité que par quelques savants autrichiens" ; peutêtre cette somme a-t-elle été complétée par d'autres voies ${ }^{80}$. Pour les deux années suivantes, Rey obtient du MIP $14000 \mathrm{~F}$ et $12000 \mathrm{~F}\left(\operatorname{sur} 75000 \mathrm{~F}\right.$ ) ${ }^{81}$, complétées par $2000 \mathrm{~F}$ de la Société française des fouilles archéologiques (SFFA) et de $6000 \mathrm{~F}$ de la Fondation Piot (AIBL) ${ }^{82}$; puis en $19269000 \mathrm{~F}$ du MIP, auxquels s'ajoute l'aide de l'Académie ${ }^{83}$. Le montage financier est identique à celui de la DAFA, qui reçoit en 1923, outre l'argent du MIP, 25000 F de l'Académie et pour 192420000 de la société savante ${ }^{84}$.

La Fondation Piot avait, durant la Guerre, déjà ainsi apporté sa part au travail collectif du SAAO, en association avec l'ÉFA et le MIP ${ }^{85}$. Pour 1924-1925, la commission de la Fondation est composée de 8 membres, parmi lesquels on retrouve Fougères, Pottier, Haussoullier, ainsi que les Athéniens Charles Diehl et Théodore Reinach et les chartistes Henry Omont et Paul Durrieu $^{86}$. C'est pour cette aide accordée par l'Académie que Léon Rey décide dans les premières années de "déposer à la bibliothèque de l'Institut de France, une étude plus complète des ruines découvertes", comme il l'avait fait pour des aquarelles et dessins du SAAO en

78 Attestée en août 1923 : AN, F17-17262 : note du MIP s.d. [août 1923]. Sa composition n'est pas connue pour 1923-1924 ; en 1925, elle comprend : Godart président, et les scientifiques Bourcart, Paul Boyer (Langues'O), Cagnat, Diehl, Fougères, Pottier, S. Reinach, Rey (Albania II, 1927, 11).

79 Rama 2012, 18 ; AEFA, Fonds C. Picard 2, 3 : carnet "Chronique personnelle" à attribuer à Fougères ; AEFA, MACED 1-1918 et MACED 1-1917-1918.

80 AN, F17-17225 : séance du 8 mars 1923. Dans celle du 17 octobre 1923, Coville évoque un budget total d'une 4oaine de mille francs", mais on ne trouve pas trace (ni pour l'AIBL ni pour la SFFA, voir infra) d'autres crédits pour 1923 .

$81 \quad$ AN, F17-17225 : séances des 6 févr. 1924 et 26 mars 1925.

82 BSFFA, 6, 1924-1925, 51 (séance du 18 mars 1924) et 64. CRAI 1924, 97 (s. du 14 mars 1924) et 1925, 150 (s. du 8 mai 1925). Rey 1925, 9 .

83 AN, F17-17225 : séance du 20 mars 1926. Des financements du même ordre sont encore attestés pour 1927 et 1928.

84 Fenet 2010, 333-334, 344, 427.

85 Homolle 1919.

86 CRAI 1923, 448 (séance du 28 déc. 1923) et 1924, 12 (s. du 11 janv. 1924). 
$1922^{87}$. Quant à la Société française des fouilles archéologiques, association créée en 1904, elle est alors en effet présidée par le chartiste et académicien Ernest Babelon (auquel succède, après sa mort le 3 janvier 1924, Homolle) et comprend notamment, parmi les 29 membres du Comité central tous impliqués dans l'archéologie française menée en Grèce et en Orient (Turquie, Égypte, Syrie...), les trois Athéniens déjà cités, membres de la Commission des fouilles au MIP, ainsi que Diehl, le chartiste Eugène Lefèvre-Pontalis, les mécènes de fouilles le duc de Loubat et le baron Edmond de Rothschild ${ }^{88}$. Le soutien de Babelon "à l'exploration archéologique de l'Albanie de la Syrie, de l'Afghanistan" est rappelé lors de ses obsèques et le Bulletin de la Société de 1923 se réjouit de cette opportunité ouverte au Nord de la Grèce. En échange de ces bons procédés, Rey - comme l'a fait Foucher - transmet à la Société un rapport sur sa mission et ses découvertes ${ }^{89}$.

Rey conçoit sa tâche en Albanie comme une extension de l'œuvre du SAAO durant la guerre : pour lui, elle entre dans le cadre de l'aide accordée au "peuple albanais, devenu libre" par "les Nations Alliées, qui furent les ouvrières de son indépendance" ${ }^{\circ}$. En tant que mission de "propagande", elle se doit d'inclure des conférences archéologiques aussi bien que de culture française (Versailles ou l'art ogival) ${ }^{91}$. Le premier projet de budget élaboré par Léon Rey comprend d'ailleurs parmi les dépenses envisagées une ligne "propagande dans la presse albanaise" ${ }^{2}$. Dans le même but (“il n'existe pas de revue consacrée à l'Albanie, la place était à prendre et nous l'avons prise" ${ }^{93}$ ), les résultats de la mission sont publiés dans une revue créée ad hoc, en langue française avec des résumés en albanais : Albania. Cahiers d'archéologie, d'histoire de l'art en Albanie et dans les Balkans ${ }^{94}$. Pensé dès 1923 , le premier volume paraît en 1925 ; en continuité avec l'œuvre passée, il inclut également des articles concernant les fouilles menées dans les Balkans dans le cadre du SAAO.

Suite à la ratification tardive de la convention, la première mission est courte (du 21 septembre au 31 octobre 1923) : elle consiste en une étude topographique et des relevés photographiques de Durazzo, avant quelques "sondages d'exploration". Dans la manière de mener ces recherches, se devine l'expérience de l'ancien membre du SAAO ${ }^{95}$ : observations du terrain dans et en dehors de la ville, prospection avec une attention particulière portée en particulier aux "entonnoirs creusés par les bombes" et aux terrains mis à nu par les maisons détruites, recherche de "renseignements" auprès des habitants et repérage du matériel "transporté" par des particuliers dans leur propriété. De même, le travail de Rey sur les remparts rappelle celui effectué par le SAAO sur ceux de Salonique. En chartiste, il pense à compléter les recherches sur le terrain par des dépouillements d'archives médiévales en Italie. L'archéologue prend en considération le moindre fragment, même s'il ne le juge pas

89 Respectivement BSSFA, 6, 1924-1925, 39 ; ibidem 5, 1923-1924, 135-137 ; ibidem 6, 1924-1925, 135-165.

$90 \quad$ AN, F17-17262: discours du 15 octobre 1923.

$91 \quad \mathrm{AN}, \mathrm{F}_{17-17262}$ : copie de la lettre de la légation de France à Tirana à Briand du 3 oct. 1926.

92 AN, F17-17262 : note datée 19 août 1923.

93 AN, F17-17262 : rapport de la campagne 1923.

94 Ibid. Plus tard paraîtra un guide de l'Albanie (Rey 1930).

95 Ibid. 
digne d'un musée, prenant ainsi en compte autant la valeur scientifique de l'artefact que sa valeur esthétique : ainsi les tessons, dont il tente de déterminer le type et l'époque et qu'il compte, ou les briques. Il prend des premières mesures de protection du patrimoine, en accord avec le maire Kost Paftali, afin d'empêcher l'extraction de pierres anciennes en vue de constructions modernes. Il s'intéresse en particulier aux trouvailles fortuites faites pendant la guerre par les soldats autrichiens. L'intendance reflète également les habitudes prises pendant la Guerre. L'hébergement se fait principalement sous tentes (fig. 3). Dans les premiers temps de la mission, Rey affirme sa volonté d'un "matériel transportable", qui permette des actions archéologiques mobiles dans les régions autorisées ${ }^{96}$. Une partie de l'équipement utile est laissé sur place dans des caisses ou cantines au monastère d'Apollonia ou à l'agence consulaire de Durazzo, avec des inventaires détaillés qui montrent la valeur accordée à la moindre chose. Outre le nécessaire pour la vie quotidienne (vaisselle, pharmacie, lits picot, moustiquaires, "un casque colonial en liège"...), on y recense les pelles et pioches, le matériel à dessin et à estampage, et quelques ouvrages : dictionnaires latin, grec (ancien et moderne), allemand et anglais, "un manuel de numismatique" 97 et "deux de topographie", le Traité d'épigraphie grecque de Salomon Reinach (1885) et le Cours d'épigraphie latine de René Cagnat (Paris, $\left.1913^{4}\right)$. Est également prévu tout un équipement pour appareil photographique "13/18", c'est-à-dire à plaques de cette dimension, et pour le développement (produits chimiques, thermomètre...), l'appareil lui-même étant mentionné à l'été 1923 comme appartenant déjà à Léon Rey ${ }^{98}$. C'est une activité qu'il pratiquait déjà durant la guerre, dès son départ en no-

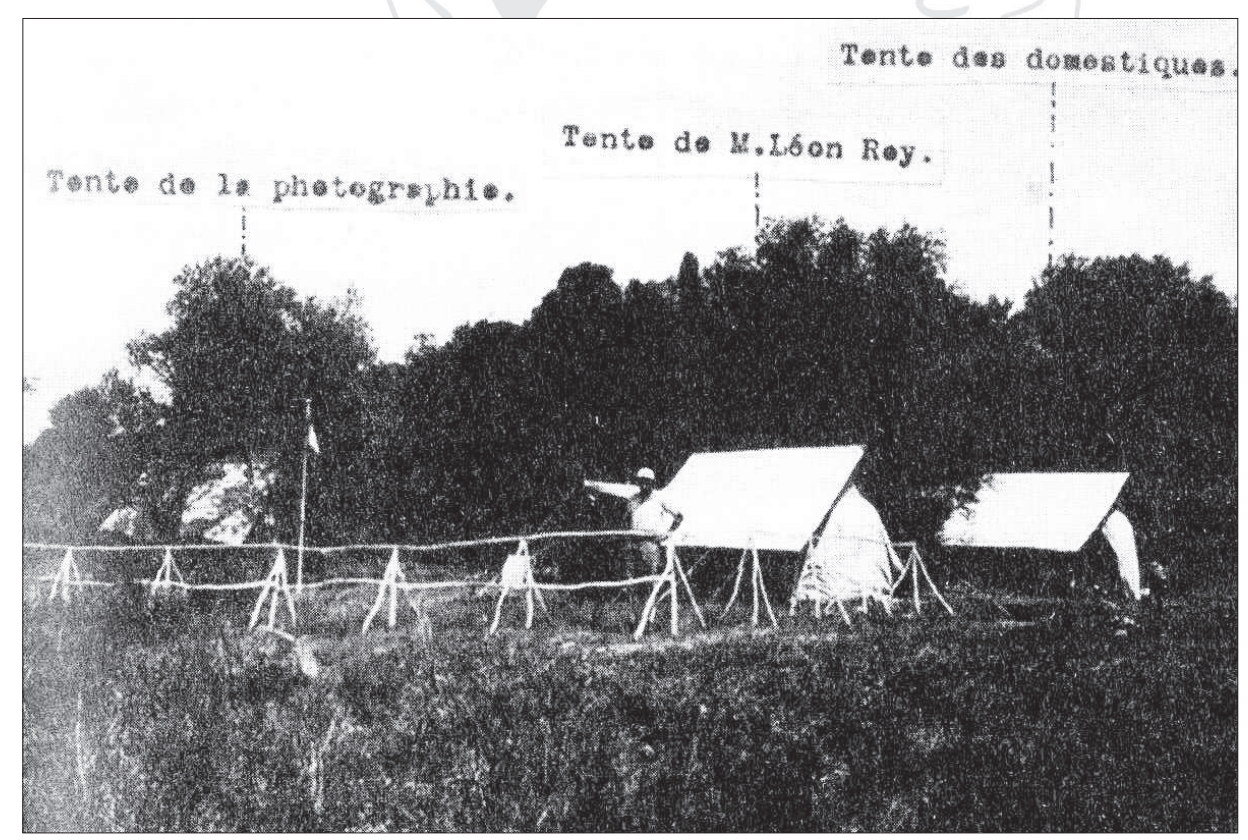

Fig. 3. Les tentes de Léon Rey à Apollonia ; d'après Rama 2010, 77.

96 Ibid. Il faudra attendre 1933 pour que soit construite la maison de fouilles sur ladite "colline 104" (maison détruite en 1992, puis quelques années plus tard rebâtie et transformée en buvette).

97 Peut-être s'agit-il du Nouveau manuel de numismatique ancienne de A. de Barthélémy (Paris, 189o)?

98 Les plaques de verre prises en Albanie sont conservées aujourd'hui aux Archives nationales de Tirana, dans le fonds Léon Rey. 
vembre 1915 vers l'Orient ${ }^{99}$. On sait l'importance que prit la photo dans les travaux archéologiques durant la Première Guerre, avec le support de la Section photographique de l'Armée $\mathrm{d}^{\prime} O \mathrm{rient}^{100}$. Ces photographies au procédé gélatino-bromure restent utilisées, comme cela a été le cas durant la Grande Guerre, par les professionnels et certains amateurs, en même temps que d'autres boîtiers de plus petit format et/ou ou à films souples ${ }^{101}$. Enfin le missionnaire n'oublie pas la signalétique nationale : deux fanions français et albanais ${ }^{102}$.

Pour 1924, à la suggestion de Rey, la Commission des missions accepte le principe de fouiller à Apollonia "un site nouveau dont il convient, sans plus tarder, que nous prenions possession"103 et que le Français avait visité seul lors de son premier voyage en Albanie en mars $1923^{104}$. Le choix s'est porté sur ce site pour sa valeur historique et archéologique, mais aussi pour les facilités qu'il offre en opposition à Durazzo : absence de ville moderne, niveaux anciens proches du sol moderne et au-dessus de la nappe phréatique, quasi vierge de toute exploration. Par la focalisation sur une fouille étendue à un seul site immense, impliquant l'abandon d'un inventaire ou carte archéologique à plus vaste échelle, puis avec la création d'un musée envisagée à Fieri en $1926^{105}$, l'on s'éloigne du programme de l'Armée d'Orient pour rejoindre celui réalisé par d'autres missions françaises en Orient. Et comme pour celles-ci, la mission albanaise se résume à un seul homme, doté de moyens limités par rapport à l'ampleur de la tâche ${ }^{106}$. Rey est seul archéologue français sur place pour mener les différents travaux, et ce seulement durant les congés d'été. En voulant s'implanter à Apollonia, Rey sait parfaitement, en décembre 1923, qu'il s'engage dans un chantier à grande échelle et à long terme, à l'image des grands sites fouillés en Grèce ou en Turquie depuis le $\mathrm{XIX}^{\mathrm{e}}$ s. Parallèlement, au même moment, Foucher est parti au nord de l'Afghanistan pour fouiller la cité de Bactres, elle aussi isolée et porteuse de grands espoirs de trouvailles. Entre les deux situations, une différence est notable : Foucher s'y rend finalement à contrecœur, presque contraint par ses autorités de tutelle à Paris, conscient, alors qu'il se trouve en Asie depuis 1918 et en Afghanistan depuis 21 mois, de l'énormité de la tâche et de la modestie de ses moyens ${ }^{107}$. En revanche, Rey ne semble pas douter de l'avenir, malgré les incertitudes relatives aux budgets futurs de la mission et l'absence même d'une légation française en Albanie. Cette confiance peut s'expliquer de diverses façons, à commencer par le manque d'expérience personnelle de Rey dans la pratique d'un grand chantier récurrent, puisque les actions qu'il a menées dans le cadre du SAAO ont toujours été ponctuelles, en même temps que par sa fréquentation avec des archéologues membres de l'ÉFA pour qui les missions de quelques mois sur un site concédé sont pour ainsi dire la norme. Par ailleurs ses expériences de l'Armée d'Orient lui ont donné l'image d'une archéologie très encadrée par les institu-

Lettres à sa sœur, citées par Rama 2012, 15-17.

Mendel 1918. Un photographe, Albinet, fut également affecté au SAAO.

Fieschi et al. 2014, 80-81 et 84-85.

102 AN, F17-17262 : listes datée des 31 août et 6 septembre 1924.

103 AN, F17-17225 : séance du 6 févr. 1924.

104 Rama 2012, 32-34.

105 AN, F17-17262 : copie de la lettre de la légation de France à Tirana à Briand du 3 oct. 1926 ; AMAE, Albanie 1 : rapport du $1^{\mathrm{er}}$ mars 1927. Le musée sera finalement inauguré à Vlora en 1936.

106 Fenet 2011b.

107 Fenet 2010, 79-82 et n ${ }^{\text {os }}$ 127, 131-132. 
tions, avec des moyens logistiques mis à disposition, en même temps qu'une assurance en terme de débrouillardise et d'action - solitaire aussi bien que collective - malgré les difficultés du contexte. Léon Rey n'est pas pour autant une tête brûlée : son projet de budget pour la première campagne de 1923, comprenant un chapitre "fonds de réserve et dépenses imprévues" (dont "accidents du travail, indemnités pour récoltes abimées, variation pour le change") ainsi que des économies (utilisation de matériel lui appartenant) ${ }^{108}$, montrent son expérience et son sens pratique. Dans tous les cas, ces choix de fouilles de grande envergure à Bactres comme à Apollonia indiquent la volonté, dans les milieux décisionnaires français de cet après-guerre, de conventions synonymes de fouilles prestigieuses limitées sur des sites phares, plutôt qu'un programme de prospections à long terme et de petites fouilles ponctuelles - ce que préconisait Foucher après plusieurs mois de présence continue sur le terrain.

La mission archéologique française en Albanie peut ainsi être définie comme une réussite hybride, issue d'un modèle perso-afghan et de l'expérience du SAAO. Sa naissance s'inscrit dans le contexte plus général de l'archéologie française de la IIIe République dans lequel des initiatives d'hommes isolés, n'appartenant pas forcément aux milieux scientifiques, sont relayées par d'autres hommes ou groupes en relation avec des tutelles académiques ou universitaires. Dans ce processus d'institutionnalisation, les conventions se négocient au cas par cas, sur le terrain plus qu'au Quai d'Orsay, avec des textes variant selon les desiderata des pays concernés et leur degré de revendication d'un patrimoine national. La lutte d'influence que souhaite mener la France dans le domaine archéologique se heurte non seulement à des oppositions externes ou internes au pays d'accueil, mais également aux faibles moyens dont elle dispose. Ces défauts sont palliés par le grand investissement personnel de fortes personnalités, comme, dans le cas albanais, Godart et Rey, qui ont produit une œuvre durable encore saluée aujourd'hui dans le "pays des Aigles".

\section{AnneXe 1.}

"Convention entre la France et l'Albanie relative aux fouilles archéologiques en Albanie" : $2^{\mathrm{e}}$ version du texte en 12 articles, à dater d'avril 1923 (AN, F/17/17262, joint au rapport de Rey du $1^{\mathrm{er}}$ mai 1923).

Les passages identiques à ceux de la convention franco-afghane (avec changement de la référence au pays concerné) sont indiqués en italiques.

Art. ${ }^{\text {er }}$.- En raison des relations de sincère amitié qui existent si heureusement et doivent se développer entre la France et l'Albanie et aussi en considération de la compétence particulière des représentants de l'archéologie en France, le Gouvernement albanais accorde au Gouvernement 
de la République française le privilège exclusif de pratiquer des fouilles archéologiques sur toute l'étendue des territoires suivants :

I. Préfecture de Scutari :

a) la sous-préfecture de Malesija e Madhe

b) la sous-préfecture de Dukagjini

c) la sous-préfecture de Puka

II. Préfecture de Durazzo :

a) la ville et tous les villages dépendant administrativement de Durazzo

b) la sous-préfecture de Kruja

III. Préfecture de Bérat :

a) la sous-préfecture de Fieri

b) la sous-préfecture de Mallakastra.

Une carte portant le tracé de ces délimitations est adjointe à la présente convention [voir fig.4].

Art. 2. Sont exclus de ce privilège tous les lieux saints et vénérés tels mosquées, églises, cimetières, etc. de même les lieux stratégiques, sauf autorisation spéciale du Gouvernement albanais. Les Délégués français chargés des fouilles devront respecter les lois, les habitudes, les coutumes et les moeurs du pays et ne rien faire qui y soit contraire.

Art. 3. Toutes les dépenses quelles qu'elles soient seront à la charge du Gouvernement de la République [française] et le Gouvernement albanais ne s'y associera en aucune façon.

Art. 4. Le Gouvernement albanais pourra adjoindre à la mission française des fouilles des savants albanais en nombre égal à celui des membres de la mission. Ces savants s'occuperont d'aider ceux-ci dans leur tâche et surveilleront avec eux les travaux des fouilles. Les frais de voyage et de séjour des délégués albanais seront à la charge du Gouvernement albanais.

Art. 5. Les délégués français auront le droit de prendre des photographies, dessins et moulages de tous les objets découverts dans les fouilles. Les moules resteront la propriété du Gouvernement albanais auquel seront en outre remis deux exemplaires de chaque photographie ainsi que deux photographies des dessins.

Art. 6. Si l'on trouve des objets de valeur en or ou en argent tels que monnaies, bijoux, etc. lesdits objets seront la propriété particulière du Gouvernement albanais. Toutefois en raison de la peine prise par les délégués français pour exécuter les fouilles, si le Gouvernement albanais décide de vendre tout ou partie desdits objets, ils auront la priorité pour les acheter au prix fixé par le Gouvernement albanais.

Art. 7. En ce qui concerne les objets en métaux non précieux (cuivre, fer, plomb, etc.) ou en pierre, terre cuite ou toute autre matière (sculptures, statues, inscriptions, etc.), la moitié de ce qui sera découvert appartiendra au Gouvernement français. Toutefois, si l'on trouve un objet unique en raison de sa forme et de sa date, il restera la propriété du Gouvernement albanais. Il en sera de 


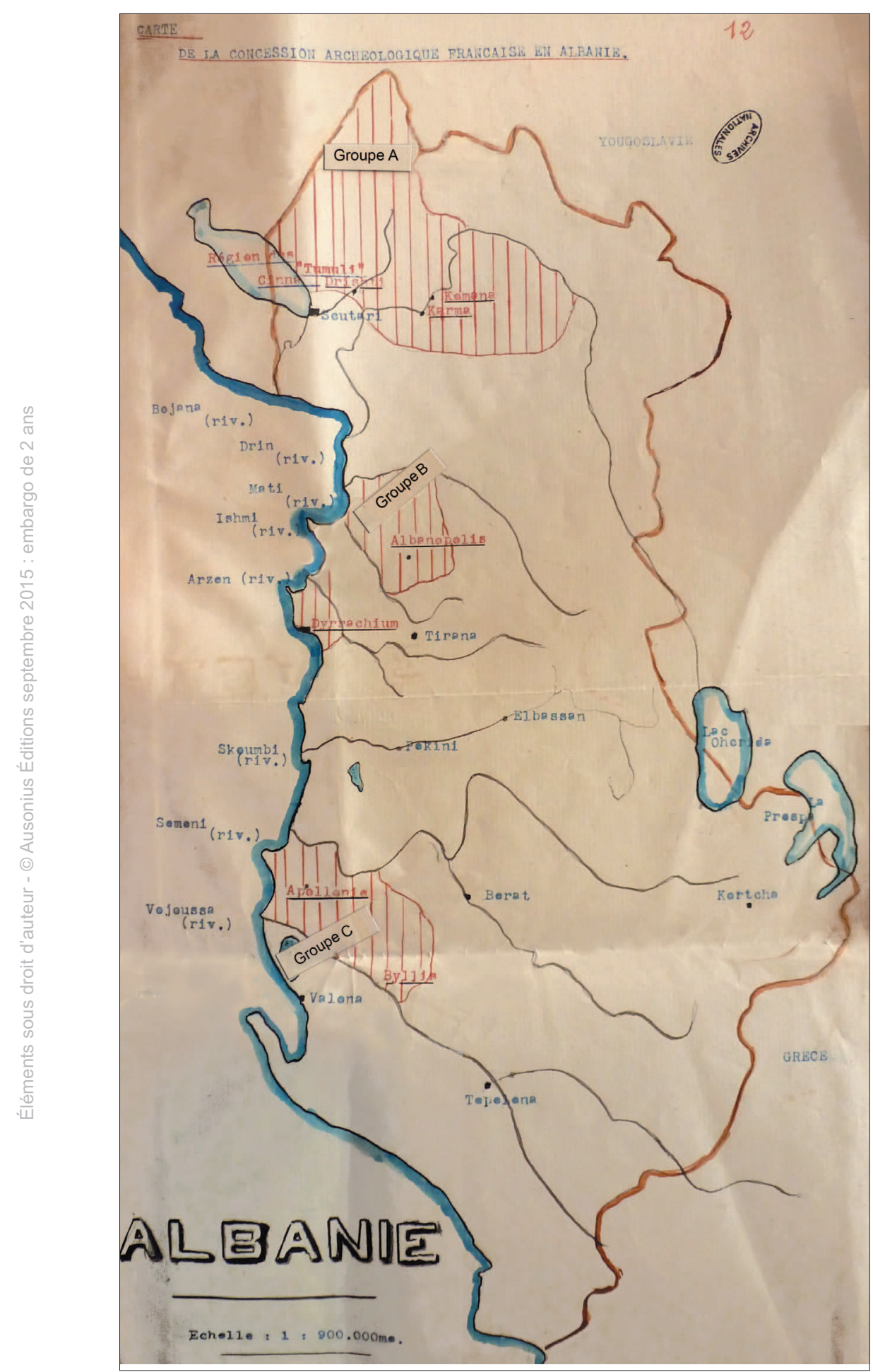

Fig. 4 Carte du projet de convention archéologique ( $7^{\text {er }}$ mai 1923). (C) Document conservé aux Archives nationales, Pierrefitte-sur-Seine, cote Fr7-17262. 
même au cas où l'on trouverait plusieurs objets formant un ensemble unique, tels, par exemple, que les différentes pièces d'un même service de table, etc.

Art. 8. Toutes les publications qui seront faites en France au sujet des travaux de la Délégation scientifique française seront offertes en cinq exemplaires au moins au Gouvernement albanais.

Tant que la Mission française n'aura pas publié le résultat de ses fouilles, le Gouvernement albanais s'engage à interdire de photographier, dessiner, ou mouler les objets en provenant déposés dans ses Musées.

Art. 9. Le Gouvernement de la République [ française] ne peut céder à aucun autre Gouvernement la concession des fouilles qu'il a ainsi obtenue du Gouvernement albanais, ni associer aucun autre Gouvernement à l'exécution de ces fouilles.

Art. 10. Le Gouvernement albanais possède tout droit de contrôle sur l'exécution de la présente Convention.

Art. 11. La présente Convention est valable pour trente ans et pourra être renouvelée d'un commun accord. Si la Délégation scientifique française venait à suspendre ses travaux (sauf en cas de force majeure tel que guerre touchant à l'État, révolution dans le pays des fouilles, etc.) pendant un an et demi, la présente convention serait annulée de plein droit et le Gouvernement albanais reprendrait la liberté de traiter, s'il le jugeait à propos, avec une autre nation.

Art. 12. La présente Convention a été rédigée en deux langues, en Albanais et en Français; l'un et l'autre texte font également autorité.

\section{AnNeXe 2.}

"Convention entre la France et l'Albanie relative aux fouilles archéologiques en Albanie"

Dernière version du texte reconstituée d'après :

- une version du texte s.d., à dater circa 20 août 1923 (AMAE, Albanie 25) ;

- les modifications portées juste avant la signature les 18-19 septembre 1923 (d'après AN, F17-17262, lettre de Rey du 22 septembre 1923);

- la version albanaise de la convention parue dans Fletorja Zyrtare (coupure de presse conservée in AN, F17-17262 : rapport de la campagne 1923).

Les passages identiques à ceux de la convention franco-afghane (avec changement de la référence au pays concerné) sont indiqués en italiques ; les variations par rapport à la version d'avril 1923 (Annexe 1) sont indiquées en gras.

Art. 1. En raison des relations de sincère amitié qui existent si heureusement et doivent se développer entre la France et l'Albanie et aussi en considération de la compétence particulière des représentants de l'archéologie en France, le Gouvernement albanais accorde au Gouvernement de la République française le privilège exclusif de pratique des fouilles archéologiques et non géologiques sur toute l'étendue du territoire suivant : 
I. Préfecture de Scutari :

a) la sous-préfecture de Malesija e Madhe

b) la sous-préfecture de Dukagjini

c) la sous-préfecture de Puka

II. Préfecture de Durazzo :

a) la ville et tous les villages dépendant administrativement de Durazzo

b) la sous-préfecture de Kruja

III. Préfecture de Bérat :

a) la sous-préfecture de Fieri

b) la sous-préfecture de Mallakastra.

Une carte portant le tracé de ces délimitations est adjointe à la présente convention [non jointe].

Art. 2. Sont exclus de ce privilège tous les lieux saints et vénérés tels mosquées, églises, cimetières, etc. de même les lieux stratégiques, sauf autorisation spéciale du Gouvernement albanais. Les Délégués français chargés des fouilles devront respecter les lois, les habitudes, les coutumes et les mours du pays et ne rien faire qui y soit contraire.

Art. 3. Toutes les dépenses quelles qu'elles soient seront à la charge du Gouvernement de la République française et le Gouvernement albanais ne s'y associera en aucune façon. De même les prix des propriétés appartenant aux particuliers et dans lesquelles seront opérées des fouilles, ou, si ces propriétés sont occupées d'une manière quelconque par la Mission française, seront payés par le Gouvernement français. La Mission française s'entendra avec le Gouvernement albanais, qui, dans ce cas, procèdera comme en matière d'expropriation pour cause d'utilité publique. [ajout du 18 septembre =] L'expropriation sera faite au nom de l'État albanais.

Art. 4. Le Gouvernement albanais pourra adjoindre à la mission française des fouilles des savants [ajout du 19 septembre =] délégués de l'État albanais en nombre égal à celui des membres de la mission. Ces savants s'occuperont d'aider ceux-ci dans leur tâche, surveilleront avec eux les travaux des fouilles [ajout du 18 septembre =] et pourront en rendre compte à leur Gouvernement au moyen de rapports, journaux ou inventaires, étant bien entendu que le Gouvernement albanais s'engage, dans la limite de l'article 8 , à ne pas communiquer, publier ni laisser publier tout, partie ou résumé de ces mémoires.

Les frais de voyage et de séjour des délégués [ajout du 19 septembre =] de l'État albanais seront à la charge du Gouvernement albanais. [ajout du 18 septembre =] Comme il est d'usage, la mission française informera le Gouvernement albanais au début de chaque campagne de travaux.

Art. 5. Les délégués français auront le droit de prendre des photographies, dessins et moulages de tous les objets découverts dans les fouilles. Les moules resteront la propriété du Gouvernement albanais auquel seront en outre remis deux exemplaires de chaque photographie ainsi que deux photographies des dessins.

Art. 6. Sil'on trouve des objets de valeur en or ou en argent [ajout du 18 septembre = ] ou en ivoire, tels que monnaies, bijoux, etc. les dits objets seront la propriété particulière du Gouvernement albanais. Toutefois en raison de la peine prise par les délégués français pour exécuter les fouilles, 
si le Gouvernement albanais décide de vendre tout ou partie desdits objets, ils auront la priorité pour les acheter au prix fixé par le Gouvernement albanais.

Art. 7. En ce qui concerne les objets en métaux non précieux (cuivre, fer, plomb, etc.) ou en pierre, terre cuite ou toute autre matière (sculptures, statues, inscriptions, etc.) on procédera de la manière suivante : si l'on trouve des objets uniques en raison de leur forme, de leur date [ajout du 18 septembre =] ou de leur auteur, ils resteront la propriété exclusive du Gouvernement albanais. Et si l'on trouve des objets en double, la moitié sera accordée au Gouvernement français.

Art. 8. Toutes les publications qui seront faites en France au sujet des travaux de la Délégation scientifique française seront offertes en cinq exemplaires au moins au Gouvernement albanais. Si le Gouvernement français ne procède pas, pendant une période de cinq ans, à la publication des travaux de la Délégation scientifique française, le Gouvernement albanais aura le droit de le faire lui-même. Tant que la Mission française n'aura pas publié le résultat de ses fouilles, le Gouvernement albanais s'engage à interdire de photographier, dessiner, ou mouler les objets provenant des fouilles déposés dans ses Musées.

Art. 9. Le Gouvernement de la République [ française] ne peut céder à aucun autre Gouvernement [ajout du 18 septembre =] ou société non française la concession des fouilles qu'il a ainsi obtenue du Gouvernement albanais ni associer aucun autre Gouvernement [ajout du 18 septembre = ] ou société non française à l'exécution de ces fouilles. [ajout du 18 septembre =] Les ouvriers employés seront albanais.

Art. 10. Tous les bâtiments construits en Albanie de la part de la Mission française, soit comme habitation, soit comme magasin, seront cédés à l'expiration de cette convention libérée de toute charge au Gouvernement albanais.

Art. 11. Le Gouvernement albanais possède tout droit de contrôle sur l'exécution de la présente Convention.

Art. 12. La mission archéologique a l'obligation de déterminer dans les territoires fixés dans le premier article des zones où elle exécutera des fouilles. Après la détermination de ces zones le reste du terrain sera libre et se trouvera à la disposition du Gouvernement albanais. Après la ratification de cette convention un délai de 5 ans est accordé à la mission pour fixer les zones en question.

Art. 13. La présente convention est valable pour trente ans et pourra être renouvelée d'un commun accord.

Art. 14. Les fouilles seront exécutées d'après les règles scientifiques, [ajout du 18 septembre = ] et il va de soi que conformément à la tradition, la mission française apportera tous ses soins à mettre en lumière les résultats de ses travaux archéologiques tant au point de vue artistique, ethnographique qu'historique.

Art. 15. Si la Délégation scientifique française [ajout du 18 septembre =] ne commençait pas les travaux dans le délai d'un an et demi à dater de la signature de la présente convention ou venait à suspendre ses travaux (sauf en cas de force majeure tel que guerre touchant à l'État, révolution dans le pays des fouilles, épidémies, etc.) pendant un an et demi la présente convention serait annulée de plein droit, et le Gouvernement albanais reprendrait la liberté de traiter, s'il le jugeait à propos, avec une autre nation. [ajout du 18 septembre =] Dans ce cas le Gouvernement de la République [française] n'aurait pas le droit de demander à l'État 
albanais aucune indemnité pour les dépenses déjà faites par la mission française et toutes les installations deviendraient la propriété de l'État albanais.

Art. 16. La présente convention a été rédigée en deux langues, en Albanais et en Français; l'un et l'autre texte font également autorité.

Art. $17^{109}$. La présente convention sera ratifiée et sera mise à exécution après ratification par le Parlement albanais.

AbrÉviATions

AÉFA = Archives de l’ÉFA (Athènes)

AIBL $=$ Académie des inscriptions et belles-lettres .

AN = Archives nationales (site de Pierrefitte-sur-Seine).

AMAE = Archives du MAE (La Courneuve).

AMAE, Albanie = AMAE, Correspondance politique et commerciale 1914-1940. Série Z : Europe 1918-1929, dossiers Albanie.

AMAE, Afgh.= AMAE, Correspondance politique et commerciale. Série E (Asie) 1918-1939, dossiers Afghanistan.

BSFFA = Bulletin de la SFFA.

ÉFA = École française d'Athènes.

MAE $=$ Ministère des Affaires étrangères (France).

MIP = Ministère de l'Instruction publique (France ; Albanie).

SAAO = Service archéologique de l'Armée d'Orient.

SFFA = Société française des fouilles archéologiques.

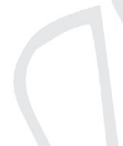

BIBLIOGRAPHIE

Adam-Veleni, P. et A. Koukouvou (2012) : Archaeology behind Battle Lines in Thessaloniki of the turbulent Years 1912-1922, Thessalonique.

Amandry, P. (1992) : "Fouilles de Delphes et pépins de Corinthe", in : Delphes 1992, 77-128.

Baldacci, A. (1917) : Itinerari albanesi (1892-1902) con un sguardo generale all'Albania e alle sue communicazioni stradali, Rome.

Blazy, S. (2004) : "Justin Godart, collectionneur et mécène", in : Wieviorka 2004, 3-9.

Bonnet, C., V. Krings et C. Valenti, éd. (2011) : Connaître l'Antiquité. Individus, réseaux, stratégies du XVIII au $X X I^{e}$ siècle, Rennes.

Bourcart, J. (1921) : L'Albanie et les Albanais, Paris.

- (1922) : Contribution à la géographie et à la géologie de l'Albanie moyenne, Paris.

Cabanes, P. (1994) : L'Albanie, le pays des aigles, Aix-en-Provence [compte-rendu par Fenet, A. (1995), Revue européenne d'histoire, 2, 111-112].

— (1996) : "L'École française d'Athènes en Épire et en Albanie", $B C H, 120-121,397-403$.

Clayer, N. (2012) : "Carl Patsch et le Musée national de Tirana (1922-1925). Construction nationale et expertise muséologique", Archéologies méditerranéennes, Revue germanique internationale, 16, 91-104.

Dassios, P. (1992) : “Les péripéties de la convention vues de Grèce”, in : Delphes 1992, 129-143.

109 Cet article ne figure pas dans la version albanaise ; en échange, dans Fletorja zyrtare, le texte est suivi de la mention du vote du Parlement albanais ( $\mathrm{n}^{\circ} 525$ à la date du 29 septembre 1923). 
Delphes (1992): La redécouverte de Delphes, Athènes-Paris.

Dimo, V., P. Lenhardt et F. Quantin, éd. (2007) : Apollonia d'Illyrie 1. Atlas archéologique et historique, Collection de l'EFR 391, Athènes-Rome.

Dimo, V., F. Quantin et B. Vrekaj (2007) : "Histoire des recherches archéologiques à Apollonia”, in : Dimo et al. $2007,25-38$.

EGA. 2 (1998) : voir “Bibliographie d'Ève Gran-Aymerich”, supra, p. 19.

EGA. 65 (2000) : voir "Bibliographie d'Ève Gran-Aymerich”, supra, p. 24.

Estournelles de Constant, P. d' (1922) : "Préface”, in : Godart 1922, 1-26.

Farnoux, A. (2012) : "Archaeology and the Armée d'Orient”, in : Adam-Veleni \& Koukouvou 2012, 82-89.

Fenet A. (2010) : Documents d'archéologie militante. La mission Foucher en Afghanistan (1922-1925), Mémoires de l'AIBL 42, Paris.

— (2011a) : "L'École française d'Athènes et la Délégation archéologique française en Afghanistan : hellénistes et indianistes unis pour une même cause (1922-1924)", in : Bonnet et al. 2011, 121-139.

— (2011b) : "Perse, Afghanistan, Extrême-Orient. Politiques archéologiques françaises en Orient dans le premier quart du $\mathrm{xx}^{\mathrm{e}}$ siècle, d'après les archives de la Société Asiatique”, in : Galitzine-Loumpet et al. 2011, 60-64.

- (2013) : "Paris-Athènes-Constantinople. Rivalités et collaborations archéologiques en Méditerranée orientale à l'aube du XX ${ }^{\mathrm{e}}$ siècle", in : Poulain et al. 2013, 43-55.

— (2014) : "Ruines et archéologie. Le front, un terrain culturel ?”, in : Vu du front 2014, 59-63.

Fenet A., F. Quantin, A. Skenderaj et B. Vrekaj (2007) : "Bibliographie générale”, in : Dimo et al. 2007, xvii-xxxi.

Fieschi, C., C. Joschke et A. Petiteau (2014) : "Photographier la guerre en amateur”, in : Vu du front 2014, 78-88.

Frank, R. (2004) : “Missions dans les Balkans”, in : Wieviorka 2004, 207-216.

Galitzine-Loumpet, A., S. Gorshenina et C. Rapin, éd. (2011) : Archéologie(s) en situation coloniale 1 : Paradigmes et situations comparées, Les Nouvelles de l'archéologie, 126, décembre.

Gilkes, O. J. (2003-2004) : “The rivals ? Leon Rey, Luigi Ugolini and their predecessors”, in : Mémoire Rey 20032004, 47-57.

Godart, J. (1922) : L'Albanie en 1921. Préface de M. d'Estournelles de Constant, sénateur, Paris.

- (2009) : Ditaret e mia (Mes journaux), Tirana.

Homolle, T. (1919) : "Rapport sur les travaux des Écoles françaises d'Athènes et de Rome pendant l'année 1917-8", CRAI, 162-180.

Jamot, P. (1933) : "Deschamps (Gaston)", Annuaire de l'Association amicale de secours des anciens élèves de l'École normale supérieure, 49-53.

Jolly, J., éd. (1960-1977) : Dictionnaire des parlementaires français. Notices biographiques sur les ministres, sénateurs et députés français de 1889 à 1940, Paris, 8 vol.

Marc, J.-Y. (1997) : “L'archéologie française en Thrace”, in : Thrace ancienne 1997, II, 473-504.

Mémoire Rey (2003-2004) : Kushtuar Leon Rey / À la mémoire de Léon Rey, Iliria, actes de colloque, Tirana 14 mai 2004, Tirana.

Mendel, G. (1918) : “Les travaux du Service archéologique de l'Armée française d'Orient”, CRAI, 9-17.

Nallbani, E. (2003-2004) : “Léon Rey, pionnier de l'archéologie française en Albanie”, in : Mémoire Rey 20032004, 41-46.

Olivier-Utard, F. [1997] (2003) : Politique et archéologie : Histoire de la Délégation archéologique française en Afghanistan (1922-1982), (1 ère éd. Paris 1997), Paris.

Pace, B. (1916-1920) : "Frustuli Illirici”, ASAA, 3, 286-29o.

Papa, A. (2000) : "Jacques Bourcart (1891-1965) et les fondements de la géologie alpine de l'Albanie”, Travaux du Comité français d'histoire de la géologie, 14, 135-143.

Poulain, M., F. Queyrel et G. Paquot, éd. (2013) : Éclats d'antiques. Sculpture et photographie : Gustave Mendel à Constantinople, Paris.

Rama, L. (2004) : “Justin Godart dans les Balkans : une vieille amitié”, in : Wieviorka 2004, 217-225.

— (2010) : Léon Rey ... dhe gurët filluan të flasin, [Albanie]. 
- (2012) : Léon Rey à la découverte d'Apollonie, trad. fr. de Rama 2010, Paris.

Rey, L. (1925) : “Fouilles de la Mission française à Apollonie d'Illyrie et à Durazzo (1923-1924) ”, Albania, I, 9-32.

- (1930) : Guide de l'Albanie, avec une carte des voies d'accès, une carte routière et les plans de Scutari, Tirana et Kortcha, Paris.

Thrace ancienne $(1997)=$ Thrace ancienne. Actes du $2^{e}$ symposium international des études thasiennes, vol. II, Komotini.

Vu du front (2014) : Vu du front. Représenter la Grande Guerre, catalogue de l'exposition, musée de l'Armée, Hôtel des Invalides, 15 oct. 2014-25 janvier 2015, Paris.

Wieviorka, A., éd. (2004) : Justin Godart : un homme dans son siècle (1871-1956), Paris.

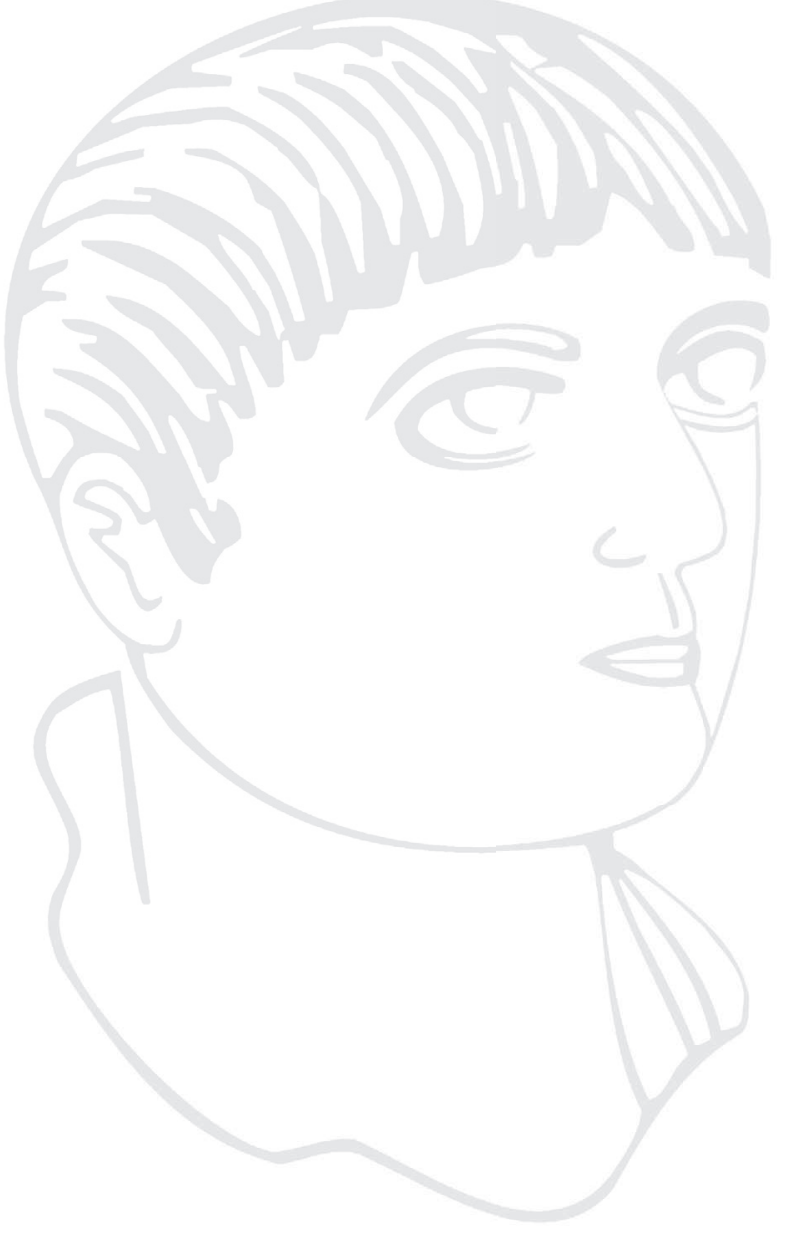

